Anna-Maria Totomanova (Sofia)

\title{
ThE SYNODIKON OF ORTHODOXY in Medieval Bulgaria
}

\footnotetext{
The Synodikon of Orthodoxy was first translated in Bulgaria by order of tsar Boril (1207-1218), who convoked a synod against the Bogomils in the capital city of Tărnovo on the $11^{\text {th }}$ of February 1211. For this reason, the Bulgarian Synodikon is usually referred to as Boril's Synodikon. Here, I am not going to delve into the circumstances that compelled a Uniate monarch to convoke an Orthodox synod $^{1}$; neither am I going to speculate whether the translation of the Synodikon was completed prior to the synod or whether the work on it only began afterwards ${ }^{2}$.
}

\footnotetext{
${ }^{1}$ First of all, it is not clear why he would resort to such a measure right at that moment: were heretics really the central domestic political issue of Boril's reign, as suggested by the unknown chronographer whose story became part of the Bulgarian Synodikon? (There sprung like some evil thorn the thrice-cursed and god-hated Bogomilism which was started then by the foulest priest Bogomil and his disciples, just as with Jannes and Jambres who once resisted Moses. And they like fierce wolves mercilessly wasted Christ's flock for which He shed his most holy blood - D 202v4-12). And if so, why had Boril's predecessors not paid any attention to this heresy? Unfortunately, the extant sources expand more on the doctrine itself than on the practices of Bogomilism, so that we are unaware of the specific political developments that led to the convening of the synod.

The very date of the synod (the year 6781, indiction 14, moon year 11, year of the solar circle 15 in the month of February (296) on the $11^{\text {th }}$ day - Friday of the Quadragesima) is given according to the Latin chronology (rather than Byzantine). This not only points to an established Uniate tradition in the royal chancellery, but also adds to the plausibility of the assumption by Bulgarian scholar Pavel Stefanov that the synod against the Bogomils might have been related to the persecutions of the Cathars in Southern France, beginning in 1208 and inspired by Pope Innocent III (1198-1216) (П. СТЕФАнов, Нов поглед кбм унията между Българската и Римската иғрква през ХІІІ в., ПКШ 5, 2001, p. 345). However, the name of Primate Basil, who was at the helm of the Bulgarian Church for almost forty years, is not on the list of the memories of the First Bulgarian Hierarchs. Possibly it was removed from the list later, following the re-establishment of the Bulgarian Patriarchate and the termination of the union in 1235; but on the other hand, tsar Kalojan, who was the prime mover behind the signing of the union, is praised therein for his many victories over the Latins and the Greeks (И. Божилов, А. ТотомановА, И. Билярски, Борилов синодик. Издание и превод, София 2010, p. 50).

${ }^{2}$ The chronographer says (P 29r4-14): After that the pious king Boril ordered the Synodikon to be translated from Greek to his language, Bulgarian. And following his orders this Holy Synod was also
} 
The first Bulgarian translation draws on the Comnenian redaction of the Constantinople version of the Synodikon ( $C$ - according to the classification of Gouillard $)^{3}$, but the extant text does not agree completely with any of the known representatives of this redaction. The earliest witness of the text (Palauzov's copy in НБКМ 289, cetera: $\mathrm{P}$ ) is dated to the end of the $14^{\text {th }}$ century and reveals strong editorial intervention, traces of which can be seen on various levels.

First of all, the editor(s) undertook a thorough linguistic revision in order to put the orthography and the grammar of the text in line with the norms of the so-called Tărnovo School, led by prominent Bulgarian spiritual leader patriarch Euthymius ${ }^{4}$.

Second, they inserted some fragments that are not to be found in the Greek original. In general, the translated part of the Synodikon (P 2r13-22r13, $\$ \$ 1-65^{5}$ ) agrees with the critical edition by Gouillard (G. 1-571), with some minor omissions, re-orderings and additions ${ }^{6}$. The sole insertion in the positive canonical part is at 5v5-8: All who came to our Orthodox faith from the unholy Armenian faith: May their memory be eternal! ${ }^{7}$, and it is obviously thematically related to the anathema upon those sharing the Armenian heresy in the added text in P 24r10-12 (\$90). This eternal memory is repeated almost literally in P 5v20-22: All who accepted Orthodox Christian faith: May their memory be eternal! The periphrasis bridges the positive and the negative canonical parts. Undoubtedly, the most important addition to the negative canonical part are the anathemas upon the Bogomils (P 13v6-15v19, $\$ \$ 39-52$ ), the source of which is the Letter of Patriarch Cosmas [Cosmas I, 1075-1081 or Cosmas II Atticus, 1146-1147] to the dearest metropolitan of Larisa in connection with the ungodly heretics ${ }^{8}$, preserved in Marcianus gr. II 74 (Coll. 1454 olim Nanianus 96), ff. 77v-79v of the $15^{\text {th }}$

entered among the Orthodox Synods to be read on the first Sunday of the holy lent like the holy fathers have taught since the very beginning of the Catholic and Apostolic Church. In the latter case, the chronographic account is presumably not completely reliable, the more so because the story of the synod apparently copies Anna Comnena's account of the trial against the Bogomils initiated by her father Alexius I Comnenus. Cf. Anne Comnène, Alexiade, XI-XV, vol. III, ed. B. LeIB, Paris 1945, ${ }^{2} 1967$, p. 218.28-228.29. Сf. И. Божилов, Византийският свят, София 2008, p. 623-628.

${ }^{3}$ J. Gouillard, Le Synodikon de l'Orthodoxie: édition et commentaire, TM 2, 1967, p. 3.

${ }^{4}$ On this matter see: А. Тотоманова, Езикбт на ХIV в. и съставът на Палаузовия сборник, Pbg 36.1, 2012, p. 24-37.

${ }^{5}$ The paragraphs are referred to according to Table 1 . The initial capital letters designate the respective manuscripts.

${ }^{6}$ The real order of the translated parts is G. $1-183,752-762,185-249,395-403,424-509,517-532$, 537-571, 752-755.

${ }^{7}$ Here and afterwards, the fragments are quoted from the English translation by М. Paneva in: И. БОЖилов, А. ТотомАновА, И. Билярски, Борилов синодик..., p. 337-377.

${ }^{8}$ J. Gouillard, Une source grecque du Synodik de Boril: la lettre du patriarche Cosmas, TM 4, 1970, p. 361-374. 
century. It is not clear how the Bulgarian translator obtained the text of the letter, but he obviously saw the richness of the information it contained and replaced the anti-Bogomil anathemas in $C$ by including the introductory part and, with some re-ordering, the text of the twelve anathemas. The latter are thematically connected with the anathema upon Basil the Doctor (P 15v20-16r3, \$53), which is also missing in the Greek Synodikon ${ }^{10}$. After the anathema on Basil the Doctor, the Bulgarian text continues according to the Constantinople redaction of the Synodikon. Then, at P 16r4-16v5 ( $\$ 54$ ), it includes only the third anathema on Eustratius of Nicaea, condemning those who deny the union of the two natures of Jesus Christ (G. 395-403), although the rubric does not mention his name explicitly ${ }^{11}$. The next 14 anathemas (P 16v6-22r13, \$\$ 54-65) follow G. 424-571, with the omission of three anathemas (G. 510-516, 533-535, 536) connected with

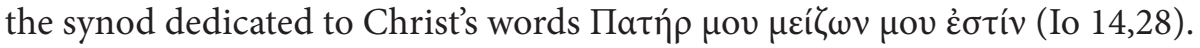

After the anathema on Gerontius of Lambe (P 9r12-20, $₫ 19$ ), seven general anti-iconoclastic anathemas have been inserted ( $\mathrm{\$} \$ 20-25)$, which in the Byzantine Synodikon occupy lines G. 752-762 in P, after the anathema on Gregory Palamas. They have been taken from the horos of the Seventh Ecumenical Council ${ }^{12}$; the first one is an anathema upon all heretics. Gouillard observes that in some $C$ version manuscripts these anathemas are included as well ${ }^{13}$. At the end of Comnenian text, our Synodikon repeats the first three anathemas (P \$\$66-68, G. 752-755), with some minor textological variations. These repetitions (not word for word, as they had already been included in the main text), however, suggest that the editors must have had at their disposal the Palaeologan version of the Synodikon. This is supported by the fact that on $\mathrm{f} .27$ (the exact place in the book cannot be identified with certainty; disagreeing with the text of version $\mathrm{P}$ ) there are anathemas upon Barlaam, Acindynus, Prochorus Cydonius, Fudul and his teacher Piropoul (P $\$ \$ 176-177)$.

The ensuing personal anathemas (P 22r21-23r16, $\$ \$ 69-78)$ were not part of the initial translation of the Synodikon either. We believe that they were also

\footnotetext{
${ }^{9}$ Cf. the opinion of Božilov in: И. Божилов, А. Тотоманова, И. Билярски, Борилов синодик..., p. 29-31.

${ }^{10}$ Where the information about the trial against Basil the Doctor was drawn from is a most interesting question. If the compiler of the Bulgarian Synodikon was also the author of the chronographic account about the synod, his source could well have been the Alexiad by Anna Comnena. But if the story was written afterwards, the information is most likely to have come from the work Panoplia Dogmatica by Euthymius Zigabenus, which was known in Slavic literary circles but has only survived in later copies of the $15^{\text {th }}-16^{\text {th }} \mathrm{cc}$. (Г. Минчев, Бориловият синодик 800 години по-късно, Pbg 35.2, 2011, p. 74-77).

${ }^{11}$ В.А. Мошин, Сербская редакция синодика в неделю православия. Анализ текстов, ВВ 16, 1959, p. 343.

12 J. Gouillard, Le Synodikon..., p. 92 (№ 308).

${ }^{13}$ Ibidem, p. 21-22.
} 
inserted by the scholars who revised the text of Boril's Synodikon to make it part of a canonical-liturgical compilation, which also included some liturgical services (all to be carried out by the patriarch) as well as the horoi of three ecumenical councils (IV, VI and VII) and of two local councils (of patriarch Menas and Tomos of Union), containing the main dogmas of Orthodoxy. They were especially selected so as to prove the need of restoration of the veneration of icons ${ }^{14}$. The eparchial metropolitans, who were in charge of performing the ritual of the Triumph of Orthodoxy, were provided with a similar type of collections of horoi of the ecumenical and local councils in Byzantium ${ }^{15}$. The linguistic evidence shows that the horoi were translated specifically for the occasion and share common orthographic and grammatical features with the revised text of the Synodikon itself. The text of the services, on the other hand, does not show any traces of editorial intervention.

The anathemas on Theodore of Pharan, Sergius and Pyrus, Peter and Paul - patriarchs of Constantinople, Honorius - pope of Rome, Cyrus of Alexandria, Macarius of Antioch and his disciple Stephen (P 23r1-7), which are missing from the Greek Synodikon, have been taken directly from the horos of the $6^{\text {th }}$ Ecumenical Council ${ }^{16}$. We believe that almost the entire list of anathematized heretics in this part was mostly drawn from the horoi of the $7^{\text {th }}$ and the $6^{\text {th }}$ ecumenical councils as well as, to a lesser extent, from the horos of the $4^{\text {th }}$ Ecumenical council and of the council of patriarch Menas ${ }^{17}$. Only five out of the 30 names of Byzantine heresiarchs are missing from the above-mentioned horoi: Symeon Magus, Kukuvrik Manent, Eusebius, Naucratius and Jacob. Since we have no data on the dissemination of the horos of the council of 843 (the origin of which has not been fully clarified by Byzantologists ${ }^{18}$ ) in the Slavic language, and bearing in mind that it does not contain the names of Eusebius, Jacob and Naucratius, it follows that the missing five names - including the names of Symeon Magus and Mani - have probably come from a different source ${ }^{19}$.

\footnotetext{
${ }^{14}$ For details on the content of the collection, which also contains the Greek text of the horoi and four noted Greek chants, vide: И. Божилов, А. ТотомАновА, И. Билярски, Борилов синодик..., p. 58-62.

${ }^{15}$ Cf. also A. Тотоманова, Синодик иаря Борила в сборнике Палаузова (НБКМ № 289), [in:] XХI ежегодная богословская конференция. Церковно-историческия исследования в контексте современной науки, Москва 2011, р. 165-166.

${ }^{16}$ For the coinciding texts vide: А. Тотоманова, Синодик изаря Борила..., p. 167.

${ }^{17}$ Ibidem, p. 170-171. See there also our polemics with Božilov, who considers the horos of the Council of 843 published by J. Gouillard (Le Synodikon..., app. 1, p. 293-298) to be the main source of this part.

${ }^{18}$ J. Gouillard, Le Synodikon..., app. 1, p. 291.

${ }^{19}$ On the mocking nickname for Mani, who is called Kukuvrik in the Bulgarian Synodikon, vide: А. Тотоманова, За една парономасия в Бориловия синодик, [in:] Gловеса пютуюднағ. Юбилеен сборник в чест на проф. Иван Буюклиев, еd. А. ТотомановА, Р. ВлАховА-РуйковА, София 2012, p. 36-43.
} 
Upon all the heretics: Anathema at P 23r17 ( $\$ 79)$ opens the next part, which contains 26 rubrics with anathemas and praises (P 23r17-25v17, \$\$ 80-104). Seventeen of them are anathemas upon basic Bogomil beliefs and practices; these generally repeat the 12 anti-Bogomil anathemas (P 13v6-15v19, $\$ \$ 39-52)$ in simpler language, more accessible to the faithful. Two anathemas (P 25r20-25v8, $\$ \$ 101-102)$ curse those who devote themselves to sorcery, one (P 25v15-17, $\$ 104)$ condemns all thieves, murderers and robbers ${ }^{20}$, and the anathema at 24r10-12 ( $\mathrm{P} \$ 90)$ is directed against those sharing the Armenian faith. The first eternal memory is for those who renounced all heresies in the name of the Orthodox faith (P 24r6-9, § 89), the second (P 25r9-19, \$ 100) - for those who retain the Orthodox faith according to the Gospels, while the third one is for all boyars, priests and monks and all the people who piously keep their devotion to the king and to the archbishops pure and righteous. This part, which likewise has to be the result of $14^{\text {th }}$-century editing, ends with the exclamation (P 25v18-19, $\$ 105)$ : Christ is victory, Christ rules, Christ is the joy of Christian faith. God save Christian faith! ${ }^{21}$, which is to be repeated thrice.

The commemorative part of Boril's Synodikon starts with a list of Byzantine rulers and their wives (P 25v20-26v20, $\$ \$ 106-109$ ). It does not completely agree with the list in the Greek Synodikon; it begins with a praise to Constantine the Great and his mother Helena (P 25v20-26r3, \$ 106) and contains the names of four rulers (Theodosius, Honorius, Theodosius II and Marcianus) which are also missing in the Greek original. Undoubtedly, the addition of the name of Marcianus is connected to his wife Pulcheria's being included in the list of empresses. Here, too, the connection between the text and the horoi can be detected, since Marcianus is mentioned repeatedly as a 'new Constantine' and Pulcheria as a 'new Helena'22. The list of Bulgarian rulers begins with the name of prince Boris ${ }^{23}$ (D 201v16-19), who made Bulgaria part of the Christian world; his praise is, in a way, a reminiscence of the praise to Constantine the Great. As regards the names of the rulers of the First and the Second Bulgarian Tsardoms, three rubrics (D 202r5-202r17, $\$ \$ 88-90)$ of the commemorative part are devoted to the memory of the Holy Brothers Cyril and Methodius, who translated the Holy Scripture into Bulgarian, and to their disciples Clement, Gorazd, Sava and Nahum. The commemorative part comprises two chronographic accounts: an account of the synod against the

\footnotetext{
${ }^{20}$ We find a similar anathema on f. $27 \mathrm{r} 1-8(\mathrm{P} \$ 175)$, before the anathemas upon Acindynus and Barlaam.

${ }^{21}$ The exclamation is strongly reminiscent of the refrain of the laudes regiae (Christus vincit, Christus regnat, Christus imperat) and comes from the Byzantine ceremonial. I feel obliged to express my gratitude to my colleague Michael Želtov, who located the phrase in Constantine Porphyrogenitus' De Ceremoniis.

${ }^{22}$ А. Тотоманова, Синодик изаря Борила..., p. 168-199.

${ }^{23}$ The list is restored according to the so-called Drinov copy (cetera: D), which shares this part with $\mathrm{P}$; see below.
} 
Bogomils convened by tsar Boril (P 29r4-30v2, $\$ \$ 110-112)^{24}$ and an account about the re-establishment of the Bulgarian Patriarchate in 1235 under tsar Ivan Asen II $(\mathrm{P} 30 \mathrm{r} 3-32 \mathrm{v} 10)^{25}$. The list of tsarinas (P 34r1-35v3, \$\$ 117-128) includes only the names from the times of the Second Bulgarian Tsardom; it ends with the wife and children of the last Bulgarian monarch, Ivan Šišman, whose name is missing on the list of rulers ${ }^{26}$. It is followed by the names of servants to the royal family ${ }^{27}$ (P 35v4-33r16, \$\$ 129-137), an incomplete commemorative list of Bulgarian patriarchs $^{28}$, and a list of metropolitans (P 37r1-39r21, $\left.\$ \$ 150-155\right)$ containing a total of 140 names. The eternal memory of all spiritual leaders of the Bulgarian nation (P 39v1 $-4, \$ 165)$ is logically followed by a praise to all boyars (P 39v5-9, $\$ 165)$ and a list of names of so far unidentified persons.

It was believed until recently that the text of Boril's Synodikon has another extant witness D, included in a Damaskin compilation from the $16^{\text {th }}$ century (НБКМ 432) ${ }^{29}$. In fact, D contains the most important insertions and additions of the first translation: the anti-Bogomil anathemas (D \$\$ 47-59) drawn from the Letter of Patriarch Cosmas and the anathema on Basil the Doctor (D \$ 60) following the anathemas on John Italus ( $\mathrm{D} \$ 45$ ) and Nilus Cabasilas ( $\mathrm{D} \$ 46$ ), coinciding with $\mathrm{P} \$ \$ 36-37$. The second one, in fact, repeats $\mathrm{D} \$ 36$ above, but in a different redaction. The text after these anathemas (D $\$ \$ 61-67$ ) agrees completely with the text of Boril's translation and corresponds to G. 395-403, 424-471, 537ff. The anathema on Constantine of Bulgaria, metropolitan of Cercyra (D \$67) lacks the ending due to the loss of some folia, but the ensuing text (D $\$ \$ 68-81$ ) comprises fifteen out of the 26 anathemas preceding the list of rulers in Boril's Synodikon. We do not know how many folia are missing, but it seems that $\mathrm{D}$ might have contained the personal anathemas of $\mathrm{P}$ as well. Drinov's text also includes the final exclamation Christ is victory, Christ rules, Christ is the joy of Christian faith. God

\footnotetext{
${ }^{24}$ The above-mentioned rubrics, as well as the beginning of the narrative about the Synod in 1211, did not survive in Palauzov's copy and were restored according to D; see below.

${ }^{25}$ In all likelihood, these accounts, too, were added during the final redaction of the text in the $14^{\text {th }}$ century. Cf. А. Тотоманова, Езикът..., p. 35-36. The end of the list of rulers was restored according to the other witness.

${ }^{26}$ On the reasons for this and other omissions in this list, vide: И. Божилов, А. ТотомАновА, И. Билярски, Борилов синодик.., p. 48-50.

${ }^{27}$ At P 35v4-7 ( $\left.\$ 129-130\right)$, there is a later addition connected with the use of the book in Wallachia after Bulgaria's fall under Ottoman rule. A similar addition with the names of two Moldavian rulers of the $16^{\text {th }}$ century is to be found at P 40r8-13 ( $\left.\$ 172-174\right)$. Concerning these additions cf. И. Божилов, А. ТотомАновА, И. Билярски, Борилов синодик..., p. 376, fn. 197-199.

${ }^{28}$ It is preceded by two rubrics ( $\left.\mathrm{P} 36 \mathrm{r} 1-5, \$ 138-139\right)$ containing the names of two ecumenical patriarchs of the end of the $13^{\text {th }}$ century. Cf. И. Божилов, А. ТотомАновА, И. Билярски, Борилов синодик..., p. 375, fn. 186, 187.

${ }^{29}$ Both witnesses are kept in the St.St. Cyril and Methodius National Library in Sofia and bear the names of prominent historians Spiridon Palauzov (1818-1872) and Marin Drinov (1838-1906), who discovered the respective manuscripts and were the first to study them.
} 
save Christian faith! ( $\mathrm{D} \$ 82$ ), the whole list of Byzantine rulers, and parts of the list of Bulgarian rulers, comprising the historical accounts, cf. P $\$ \$ 106-116$ and $\mathrm{D} \$ \$ 83-93^{30}$.

The rest of the text of Drinov's Synodikon, as compared to the text of Palauzov's copy, shows numerous differences in terms of word order, Stylistics and lexis ${ }^{31}$. A hand of the $17^{\text {th }}$ century transmitted to us a part of the lost beginning of $\mathrm{P}$, having copied the fading first rows on the wooden book cover:

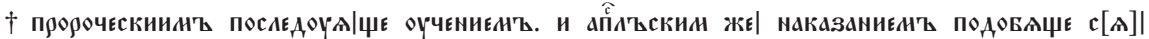

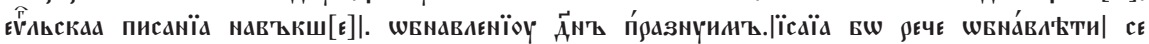

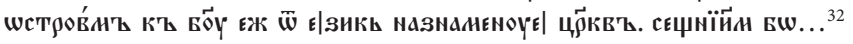

The parallel text in D 184r4-8 reads:

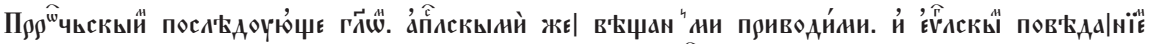

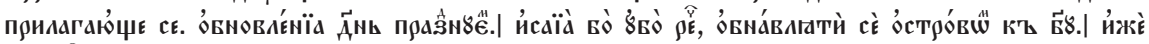

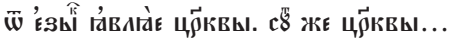

Once again, this proves that the initial part of $\mathrm{D}$ must have come from a different redaction.

In fact, the initial part of Drinov's Synodikon shares some important features with the text of the South-Slavonic Synodikon kept in Romania (BAR MS. SL. 307, cetera: $\mathrm{R})$, which unequivocally belongs to the Palaeologan version of the Synodikon ( $P$ according to Guillard). The fragments $\$ \$ 1-42$ (G. 1-170, 395-471, 171-249, 479-532) are common to both D and R; unlike P, they contain the memory of St. Andrew of Crete in the positive part (D 188r8-9 and R 6r24-25) and an anathema connected to the problem of incarnation ( $\$ \$ 16)$ in the negative part. The latter is also missing in P and G., but was included in both Greek and Slavonic printed triodia ${ }^{33}$. The inclusion of eternal memory to both St. Theodore Studites and St. Theophanes the Confessor in R 6v12-13, missing from the Greek Synodikon as well as from $\mathrm{P}$ and the printed triodia ${ }^{34}$, is the only structural difference between $\mathrm{D}$ and $\mathrm{R}$ in this initial part. At the same time, this part lacks the

\footnotetext{
${ }^{30}$ Both manuscripts are severely damaged in this part (cf. the comments on $\mathrm{P} \$ 10$ and $\mathrm{D} \$ 93$; $\mathrm{P} \$ 116$ and $\mathrm{D} \$ 94, \mathrm{D} \$ 103$ ), but the extant texts complement each other and allow us to presume that they come from a common source.

${ }^{31}$ The variant readings are duly reported in the edition: И. Божилов, А. ТотомАновА, И. БилярСки, Борилов синодик...; as well as in: Synodicum Bulgaricum 1211, ed. A. TотомаNova, [in:] The Great Councils of the Orthodox Churches. From Constantinople 861 to Moscow 2000, ed. A. Melloni, vol. I, Turnhout 2016 [= CC.COGD 4.1], p. 426-468.

${ }^{32}$ И. Божилов, А. ТотомАновА, И. Билярски, Борилов синодик.., р. 66.

${ }^{33}$ И. Билярски, Палеологовият синодик в славянски превод, София 2013, p. 27.

${ }^{34}$ Ibidem, p. 75, fn. 17.
} 
insertions typical of $\mathrm{P}$ (anti-Armenian anathemas and praises of those who came back to the Orthodoxy from the heresies, as well as the entire part drawn from the horos of the $7^{\text {th }}$ ecumenical council; G. 752-762). This means that, following the anathema on Constantine of Bulgaria, metropolitan of Cercyra ( $\$ \$ 44$ ), the text of $\mathrm{D}$ must have comprised the anathemas on Constatine's followers and on John Irenicus and by all means the above-mentioned seven general anti-iconoclastic anathemas. The idea that the so-called Drinov copy in fact represents another version of the Synodikon of Orthodoxy was first promoted by I. Biljarski and M. Tsibranska-Kostova, who noticed that - alongside structural similarities - the text in D and the Synodikon from Bucharest (R) also share some peculiar terminological features. These involve three compound words with the first part въкоупо- instead of traditional literary єднно-, such аs в'ъкоүповожьн'ь, въькоүпославь-

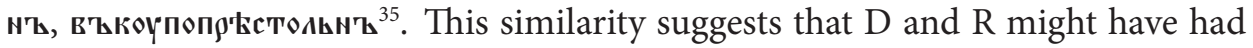
a common antigraph. In his edition of the extant text of R, Ivan Biljarski presumes that Drinov's copy belongs to the Palaeologan redaction as well ${ }^{36}$. In fact, the comparison of the extant text of Drinov's Synodikon with the other two versions $\mathrm{P}$ and $\mathrm{R}$ proves that it represents a rather mechanical compilation of the new translation (partially preserved in BAR MS. SL. 307) and the Synodikon of tsar Boril in its $14^{\text {th }}$ century version. This explains why some of the rubrics in $\mathrm{D}$ are repeated in different redactions: the anathema on Nilus Cabasilas $\mathrm{D} \$ 36$ according to redaction $P$ and $\mathrm{D} \$ 43$ - according to $C$. Cf. also $\mathrm{D} \$ \$ 17-21$ and $\mathrm{D} \$ \$ 61-66$, where the anathema on Michael is omitted in the text that belongs to the redaction $P$, but it is preserved in the older redaction ( $\mathrm{D} \$ 62, \mathrm{G} .424-434$ ) as well as in $\mathrm{P} \S 52$. We do not think that the anathemas on Barlaam, Acindynus and their followers (together with the following text preserved in $\mathrm{R} \$ \$ 55-65$ ) were part of $\mathrm{D}$ : it is clear that the unknown compiler of $\mathrm{D}$ relied on a Bulgarian source similar to $\mathrm{P}$ for the second part (which includes the anti-Bogomil anathemas and the list of the rulers) ${ }^{37}$. The conclusion that $\mathrm{D}$ can be divided in two parts - the initial

\footnotetext{
${ }^{35}$ The above-mentioned lexemes are to be found in the rubrics related to the dogmatic argument

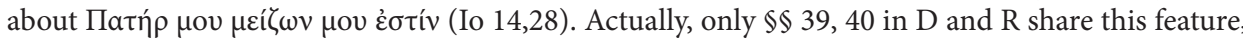
while $\mathrm{D} \$ 63$ does not agree with $\mathrm{R} \$ 44$ and displays the compound 'Алногекною, thus coinciding with P $\$ 63$ (И. БИляРСКИ, М. ЦиБРАнсКА-КостовА, За един композитен тип и за Палеологовия вариант на славянския Синодик в Неделята на православието, Pbg 36.1, 2012, p. 53-55). Cf. also p. 5 above on the coinciding parts of $\mathrm{D}$ and $\mathrm{P}$.

${ }^{36}$ И. Билярски, Палеологовият синодик..., p. 15-18, 48-50.

${ }^{37}$ In fact, the compiler replaced the anti-heretical part of $\mathrm{R}$ with its anti-Bogomil (anti-Messalian) anathemas ( $\mathrm{R} \$ 54$ ), drawn from the so-called Mount Athos Epistle, containing the decisions of the General Athonite Assembly of 1344. The text has been identified by: A. Rigo, Monaci esicasti e monaci bogomili. Le accuse di messalianismo e bogomilismo rivolte agli isicasit ed il problema dei rapporti tra esicasmo e bogomislismo, Firenze 1989 [= OV 2]. For the Greek text vide: A. Rigo, L'Assemblea generale atonita del 1344 su un gruppo di monaci bogomili (ms. Vat.Gr. 604 ff. 11r-12v), CS 5, 1984, p. 505, fig. 31-56.
} 
one Palaeologan and the second Bulgarian, based on the Comnenian redaction - leads us to some other important inferences. First, it means that the translation of the Palaeologan version of the Synodikon of Orthodoxy is an integral part of the tradition of the Bulgarian Synodikon. Second, the unknown compiler, who chose Bulgarian sources to complete his work with anti-heretical anathemas and lists of rulers, was in all likelihood addressing a Bulgarian audience and he himself must have had Bulgarian origins. In all probability, the compilation was done to meet the needs of the Bulgarian population at the end of the $14^{\text {th }}$ century. Based on certain linguistic features of D (first of all the traces of the Middle Bulgarian confusion of nasal vowel letters and the use of the letter jat for marking palatal consonants before the vowel $a$ ), Popruženko claims that the copyist of $\mathrm{D}$ used a Bulgarian antigraph ${ }^{38}$. It is worth mentioning that traces of Bulgarian Tărnovo orthography are to be found in both parts of $\mathrm{D}$ and not only in the added Bulgarian part (as one might have expected given the fact that $\mathrm{R}$ observes the Resava rules with no exceptions). Therefore, the Palaeologan version of the Synodikon obviously circulated in two orthographic recensions - those of the Resava and Tărnovo schools. This fact allows us to conjecture that the common Palaeologan antigraph of $\mathrm{D}$ and $\mathrm{R}$ must have been written in Bulgarian orthography, given the fact that Resava norms were only established by Constantine of Kostenets in $15^{\text {th }}$ century, after the fall of the Bulgarian Tsardom under Ottoman rule. If our reasoning is correct, it follows that the translation of the South-Slavic Palaeologan Synodikon must have been carried out at a literary centre that used Bulgarian orthography and was connected to Mount Athos. It is the latter location where, according to Biljarski, the Greek Palaeologan protograph arose around 1366 - after the death of Jacob, the only metropolitan of Ierisso, whose memory is mentioned the last in the list of metropolitans preserved in R $20 \mathrm{v} 15-17^{39}$. This centre cannot have been Tărnovo, where in the end of $14^{\text {th }}$ century only a revision of the early $13^{\text {th }}$ century translation was undertaken. We can only speculate whether it was the Bulgarian monastery on Mount Athos or some other monastic centre.

\footnotetext{
${ }^{38}$ М.Г. ПоПрУЖЕнко, Синодикъ ц,аря Борила, БСт 8, 1928, p. XXVIII-XXIX.

${ }^{39}$ И. Билярски, Палеологовият синодик..., р. 43-54.
} 
Table 1

Content and structure of the manuscripts

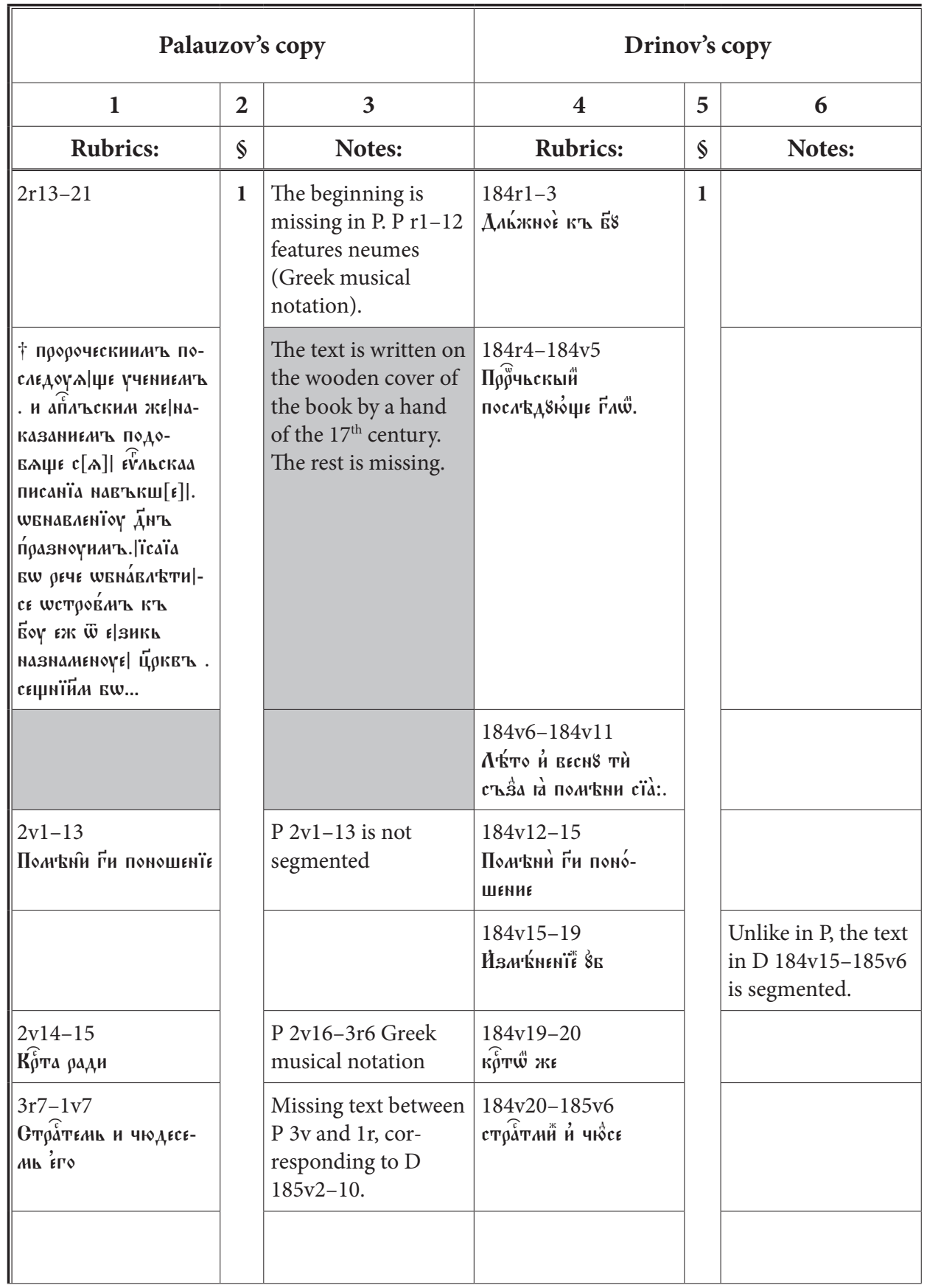


Table 1 (cont.)

\begin{tabular}{|c|c|c|c|}
\hline \multicolumn{3}{|c|}{ BAR MS. SL. 307} & \multirow{2}{*}{$\begin{array}{c}\begin{array}{c}\text { Greek } \\
\text { Synodikon }\end{array} \\
10\end{array}$} \\
\hline 7 & 8 & 9 & \\
\hline Rubrics: & $\S$ & Notes: & Rows: \\
\hline 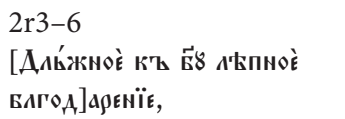 & 1 & & G. $1-3$ \\
\hline 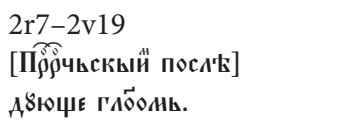 & & & G. $4-19$ \\
\hline 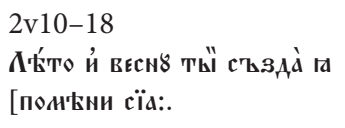 & & & G. $19-23$ \\
\hline $\begin{array}{l}\text { 2v19-22 } \\
\text { ПОАЕНЙ Г̈И ПОНО́ШЕнИЕ }\end{array}$ & & & G. $23-28$ \\
\hline 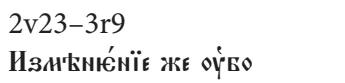 & & $\begin{array}{l}\text { Textological differences } \\
\text { in comparison with D. }\end{array}$ & \\
\hline 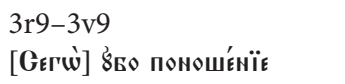 & & & G. $28-29$ \\
\hline 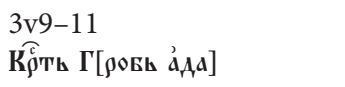 & & & G. $29-60$ \\
\hline 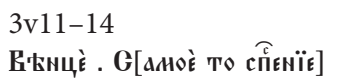 & & & \\
\hline
\end{tabular}




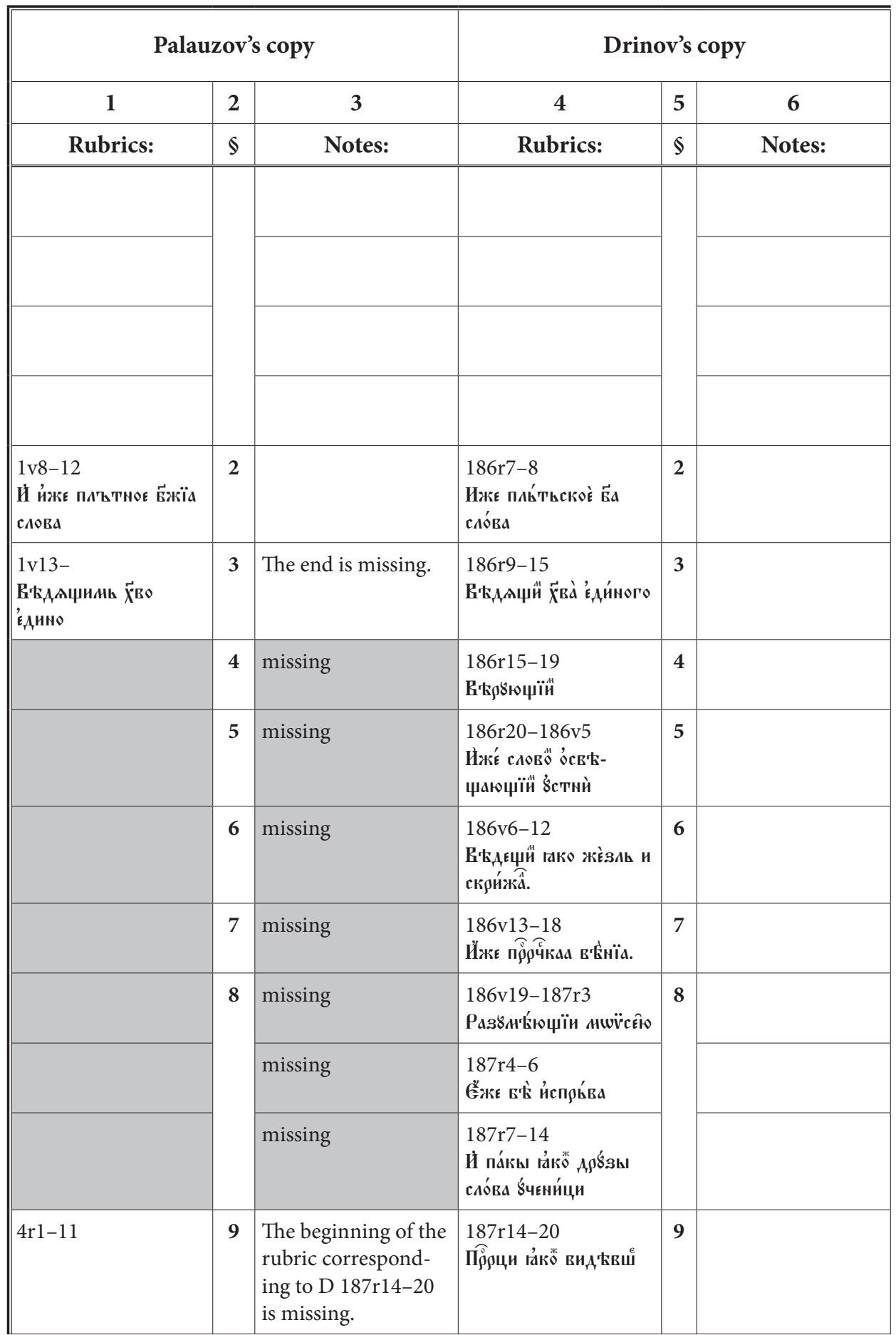


Table 1 (cont.)

\begin{tabular}{|c|c|c|c|}
\hline \multicolumn{3}{|c|}{ BAR MS. SL. 307} & \multirow{2}{*}{$\begin{array}{c}\text { Greek } \\
\text { Synodikon } \\
10\end{array}$} \\
\hline 7 & 8 & 9 & \\
\hline Rubrics: & $\$$ & Notes: & Rows: \\
\hline 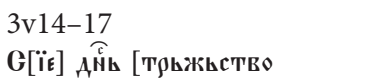 & & & \\
\hline 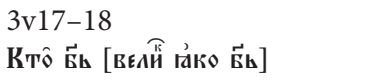 & & & \\
\hline 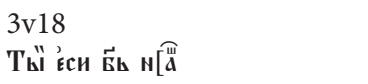 & & & \\
\hline 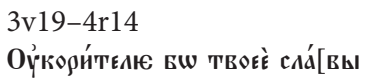 & & & \\
\hline $\begin{array}{l}\text { 4r14-18 } \\
\text { НैЖЕ ПА' }\end{array}$ & 2 & & G. $61-62$ \\
\hline 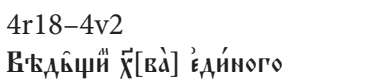 & 3 & & G. $66-67$ \\
\hline 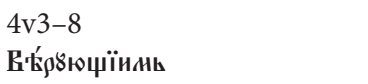 & 4 & & G. $68-71$ \\
\hline 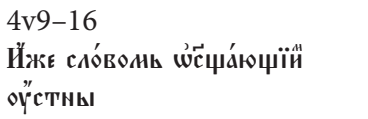 & 5 & & G. $72-75$ \\
\hline 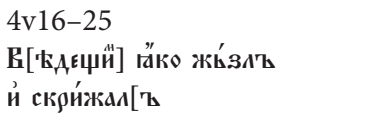 & 6 & & G. $76-81$ \\
\hline 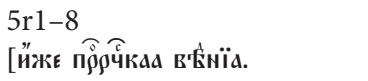 & 7 & & G. $82-86$ \\
\hline 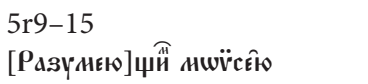 & 8 & & G. $87-90$ \\
\hline 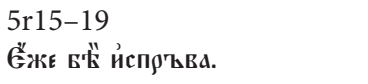 & & & G. $90-92$ \\
\hline 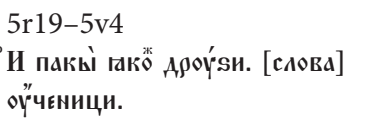 & & & G. $92-97$ \\
\hline 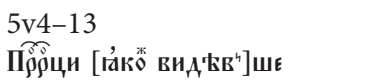 & 9 & & G. $98-102$ \\
\hline
\end{tabular}




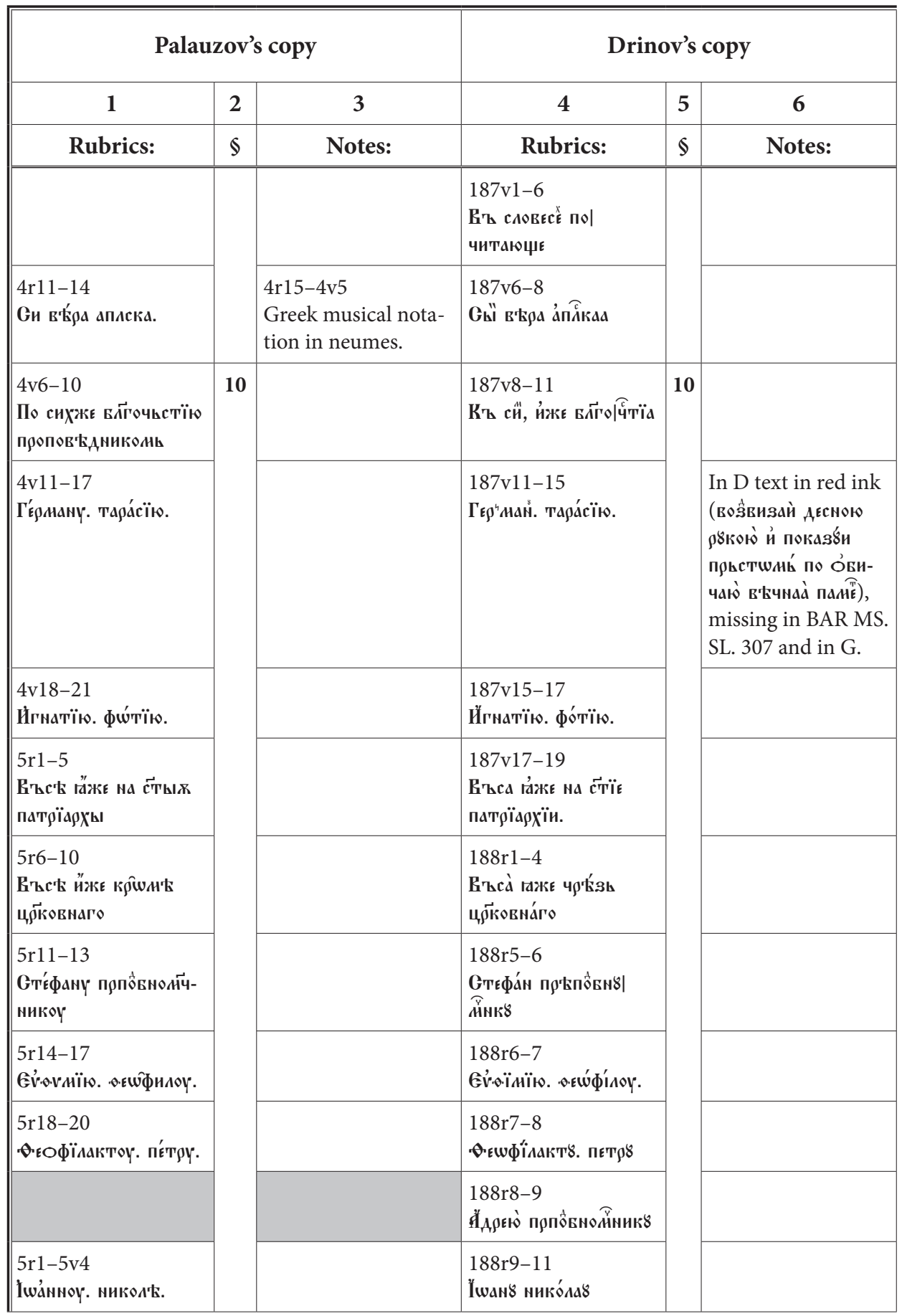


Table 1 (cont.)

\begin{tabular}{|c|c|c|c|}
\hline \multicolumn{3}{|c|}{ BAR MS. SL. 307} & Greek \\
\hline 7 & 8 & 9 & 10 \\
\hline Rubrics: & $\S$ & Notes: & Rows: \\
\hline 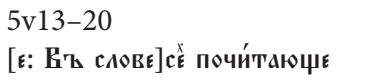 & & & G. $103-105$ \\
\hline 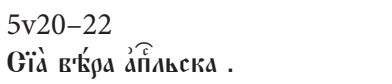 & & & G. $106-107$ \\
\hline 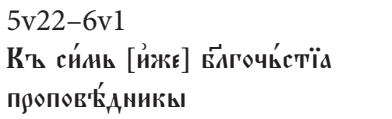 & 10 & & G. $108-109$ \\
\hline 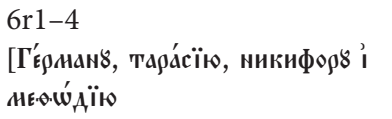 & & & G. $110-111$ \\
\hline $\begin{array}{l}\text { 6r5-7 } \\
\text { ["Нгна́тїю, фóтїю }\end{array}$ & & & G. $112-113$ \\
\hline 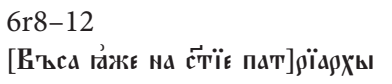 & & & G. $114-116$ \\
\hline 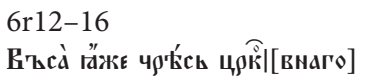 & & & G. $117-119$ \\
\hline 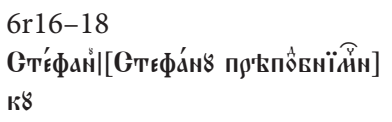 & & & G. 120 \\
\hline 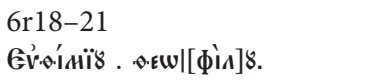 & & & G. $121-122$ \\
\hline 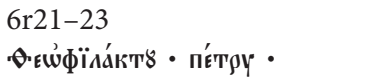 & & & G. $123-124$ \\
\hline 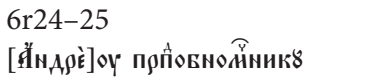 & & & \\
\hline $\begin{array}{l}\text { 6v1-4 } \\
\text { Iша[нн8, нико́лав, }\end{array}$ & & & G. $125-126$ \\
\hline
\end{tabular}




\begin{tabular}{|c|c|c|c|c|c|}
\hline \multicolumn{3}{|c|}{ Palauzov's copy } & \multicolumn{3}{|c|}{ Drinov's copy } \\
\hline 1 & 2 & 3 & 4 & 5 & 6 \\
\hline Rubrics: & $\S$ & Notes: & Rubrics: & $\$$ & Notes: \\
\hline 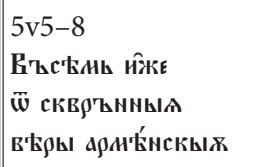 & & & & & \\
\hline 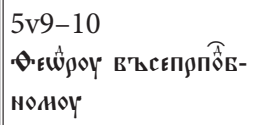 & & & 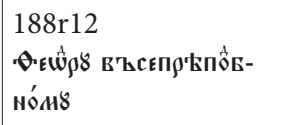 & & \\
\hline \begin{tabular}{|l}
$5 \mathrm{v} 11-12$ \\
Гсакїю чюодотворцоу
\end{tabular} & & & 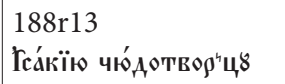 & & \\
\hline 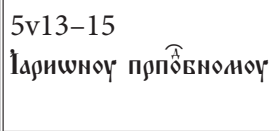 & & & 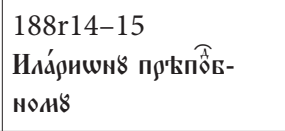 & & \\
\hline 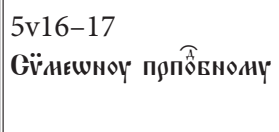 & & & 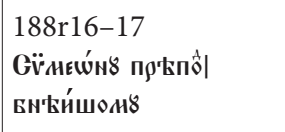 & & \\
\hline 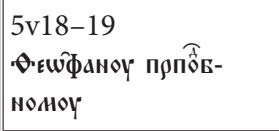 & & & 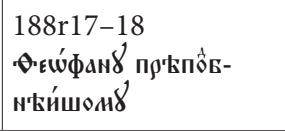 & & \\
\hline 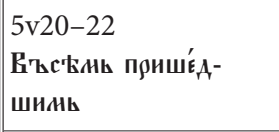 & & & & & \\
\hline 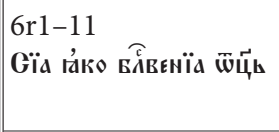 & 11 & $\begin{array}{l}\text { P 6v featuring } \\
\text { neumes and severely } \\
\text { damaged. }\end{array}$ & 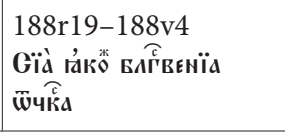 & 11 & \\
\hline $\begin{array}{l}\text { 6r11-1 } \\
\text { Словонь оүБо }\end{array}$ & & & 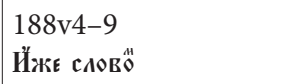 & & \\
\hline $7 \mathrm{r} 1-8$ & 12 & $\begin{array}{l}\text { The beginning } \\
\text { of the text damaged. }\end{array}$ & 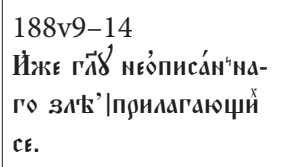 & 12 & \\
\hline 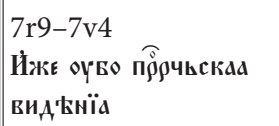 & 13 & & 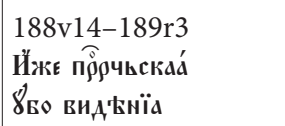 & 13 & \\
\hline
\end{tabular}


Table 1 (cont.)

\begin{tabular}{|c|c|c|c|}
\hline \multicolumn{3}{|c|}{ BAR MS. SL. 307} & \multirow{2}{*}{$\begin{array}{c}\begin{array}{c}\text { Greek } \\
\text { Synodikon }\end{array} \\
10\end{array}$} \\
\hline 7 & 8 & 9 & \\
\hline Rubrics: & $\mathfrak{S}$ & Notes: & Rows: \\
\hline 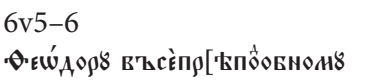 & & & G. 127 \\
\hline $\begin{array}{l}\text { 6v6-8 } \\
\text { ['T́́кїїю]| чюддотво́рц४8 }\end{array}$ & & & G. $128-129$ \\
\hline 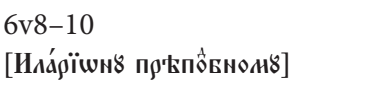 & & & G. $130-131$ \\
\hline 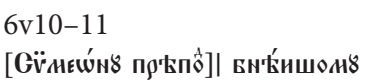 & & & G. 132 \\
\hline 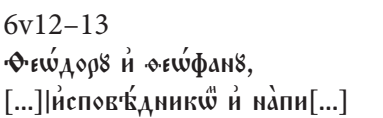 & 10 & Missing in $\mathrm{P}, \mathrm{D}$ and $\mathrm{G}$. & \\
\hline 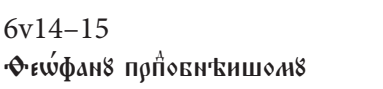 & & & G. 133 \\
\hline 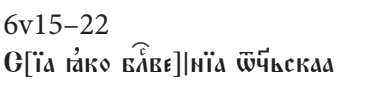 & 11 & & G. $134-137$ \\
\hline 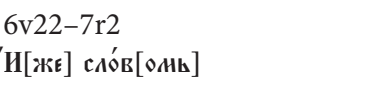 & & & G. $138-140$ \\
\hline 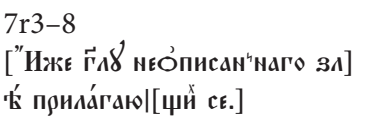 & 12 & & G. $141-144$ \\
\hline 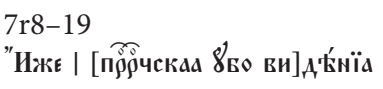 & 13 & & G. $145-150$ \\
\hline
\end{tabular}




\begin{tabular}{|c|c|c|c|c|c|}
\hline \multicolumn{3}{|c|}{ Palauzov's copy } & \multicolumn{3}{|c|}{ Drinov's copy } \\
\hline 1 & 2 & 3 & 4 & 5 & 6 \\
\hline Rubrics: & $\S$ & Notes: & Rubrics: & $\$$ & Notes: \\
\hline $\begin{array}{l}\text { 7v5-7v15 } \\
\text { Єлышжчинь г̆ }\end{array}$ & 14 & & 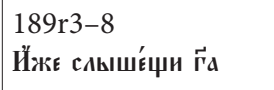 & 14 & No segmentation \\
\hline 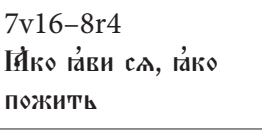 & & & 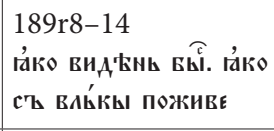 & & \\
\hline \multirow[t]{7}{*}{ 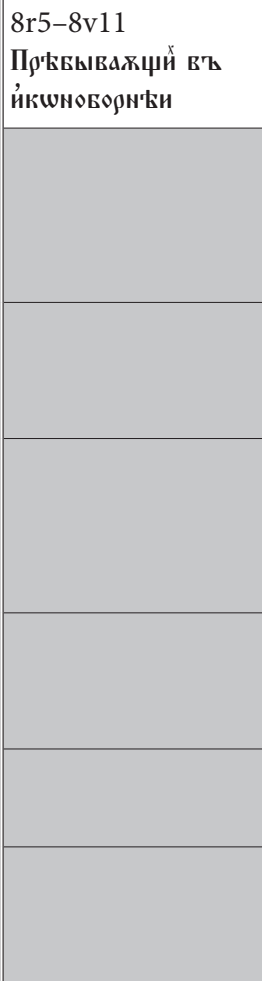 } & 15 & & 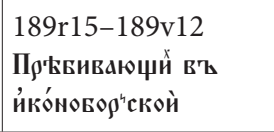 & 15 & \\
\hline & & & 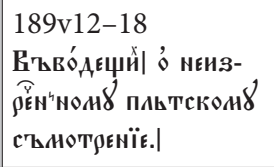 & 16 & \\
\hline & & $\begin{array}{l}\text { Compare this in } \mathrm{P} \\
16 \mathrm{r} 4-16 \mathrm{v} 5 \text { (\$ 54). }\end{array}$ & 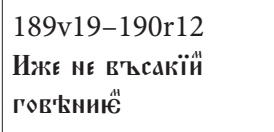 & 17 & $\begin{array}{l}\text { Repeated in D } \\
197 \mathrm{v} 4-17(\$ 61) .\end{array}$ \\
\hline & & $\begin{array}{l}\text { Compare this in } \mathrm{P} \\
17 \mathrm{r} 10-17 \mathrm{v} 5(\$ 56) \text {. }\end{array}$ & 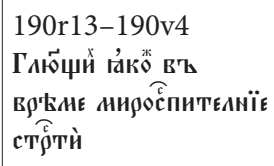 & 18 & $\begin{array}{l}\text { Repeated in D } \\
198 \mathrm{r} 12-198 \mathrm{v} 2 \\
(\$ 63) .\end{array}$ \\
\hline & & $\begin{array}{l}\text { Compare this in } \mathrm{P} \\
17 \mathrm{v} 6-16(\$ 57) \text {. }\end{array}$ & 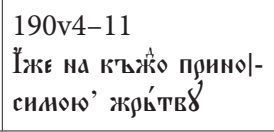 & 19 & $\begin{array}{l}\text { Repeated in D } \\
198 \mathrm{v} 3-9(\$ 64) .\end{array}$ \\
\hline & & $\begin{array}{l}\text { Compare this in } \mathrm{P} \\
17 \mathrm{v} 17-18 \mathrm{v} 6(\$ 58) .\end{array}$ & $\begin{array}{l}\text { 190v11-191r11 } \\
\text { ИैЖѐ сль'шєџй }\end{array}$ & 20 & $\begin{array}{l}\text { Repeated in D } \\
\text { 198v9-199r9 (\$ 65). }\end{array}$ \\
\hline & & $\begin{array}{l}\text { Compare this in } \mathrm{P} \\
18 \mathrm{v} 7-21(\$ 59) \text {. }\end{array}$ & 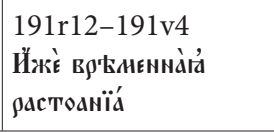 & 21 & $\begin{array}{l}\text { Repeated in D } \\
199 r 9-21(\$ 66) .\end{array}$ \\
\hline $\begin{array}{l}\text { 8v12-16 } \\
\text { фेнаста́сїа. кшстан- } \\
\text { дина }\end{array}$ & 16 & & 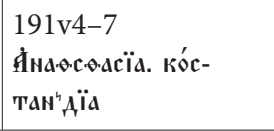 & 22 & \\
\hline 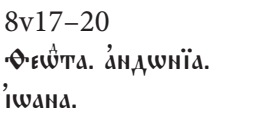 & 17 & & 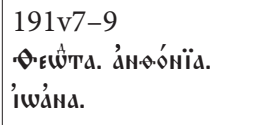 & 23 & \\
\hline $\begin{array}{l}\text { 8v21-9r11 } \\
\text { Па́вАА ЙЖЕ вЈ савАА }\end{array}$ & 18 & & $\begin{array}{l}\text { 191v9-16 } \\
\text { Па́вАА йЖЕ вา савАА }\end{array}$ & 24 & \\
\hline
\end{tabular}


Table 1 (cont.)

\begin{tabular}{|c|c|c|c|}
\hline \multicolumn{3}{|c|}{ BAR MS. SL. 307} & \multirow{2}{*}{$\begin{array}{c}\text { Greek } \\
\text { Synodikon } \\
10\end{array}$} \\
\hline 7 & 8 & 9 & \\
\hline Rubrics: & $\S$ & Notes: & Rows: \\
\hline 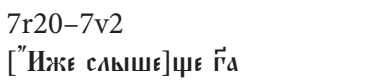 & 14 & No segmentation & G. $151-158$ \\
\hline 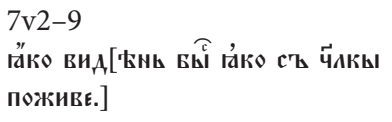 & & & \\
\hline 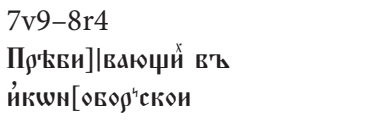 & 15 & & G. $159-170$ \\
\hline 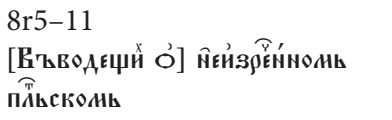 & 16 & & $\begin{array}{l}\text { The Greek text in Popruženko } \\
\text { (p. 18-19, \$16). }\end{array}$ \\
\hline 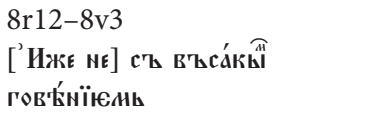 & 17 & & G. $395-403$ \\
\hline 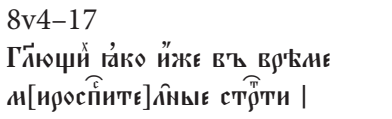 & 18 & & G. $435-443$ \\
\hline $\begin{array}{l}\text { 8v18-25 } \\
\text { Нже на к'ЪЖАО Приносин४ю } \\
\text { ж९ъте[8] }\end{array}$ & 19 & & G. $444-448$ \\
\hline $\begin{array}{l}\text { 9r1-25 } \\
\text { [”Нжє сль́́шєщй̀] }\end{array}$ & 20 & & G. $449-462$ \\
\hline 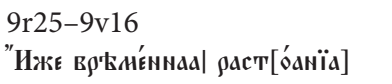 & 21 & & G. $463-471$ \\
\hline $\begin{array}{l}\text { 9v17-20 } \\
\text { Янаста́сїа, консттандй[на] }\end{array}$ & 22 & & G. $171-172$ \\
\hline 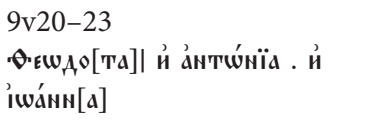 & 23 & & G. $173-174$ \\
\hline $\begin{array}{l}\text { 9v24-10r7 } \\
\text { ПавАа йЖє в'ъ са́вла }\end{array}$ & 24 & & G. $175-179$ \\
\hline
\end{tabular}




\begin{tabular}{|c|c|c|c|c|c|}
\hline \multicolumn{3}{|c|}{ Palauzov's copy } & \multicolumn{3}{|c|}{ Drinov's copy } \\
\hline 1 & 2 & 3 & 4 & 5 & 6 \\
\hline Rubrics: & $\S$ & Notes: & Rubrics: & $\mathfrak{S}$ & Notes: \\
\hline 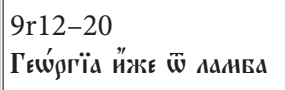 & 19 & & 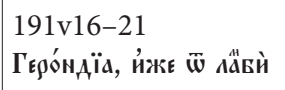 & 25 & \\
\hline 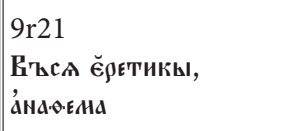 & 20 & & & & \\
\hline 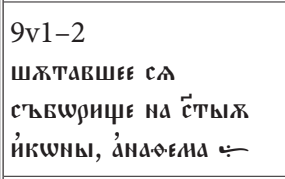 & & & & & \\
\hline 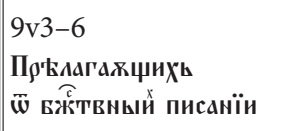 & 21 & & & & \\
\hline 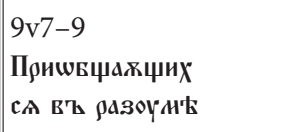 & 22 & & & & \\
\hline 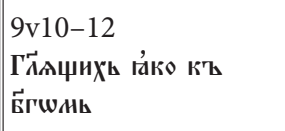 & 23 & & & & \\
\hline 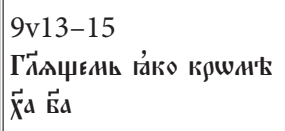 & 24 & & & & \\
\hline 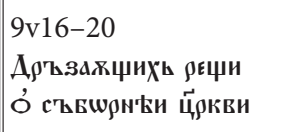 & 25 & & & & \\
\hline 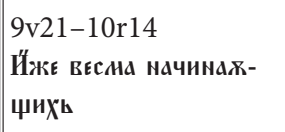 & 26 & & $\begin{array}{l}\text { 192r1-9 } \\
\text { Иैжє ш̈н8ૈ начинаю̆чеїй }\end{array}$ & 26 & \\
\hline $\begin{array}{l}\text { 10r15-10v2 } \\
\text { НैЖе Б̈ГочьстТовати }\end{array}$ & 27 & & 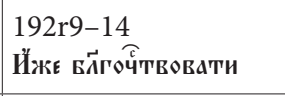 & 27 & \\
\hline $\begin{array}{l}10 \mathrm{v} 3-13 \\
\text { Иैжє Боүлд в'ьн'Еш- } \\
\text { ниХь филОсОфь }\end{array}$ & 28 & & 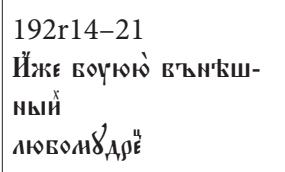 & 28 & \\
\hline $\begin{array}{l}\text { 10v14-11r4 } \\
\text { ИેЖЕ ВЕЧЕСТ'ҚВНОЕ } \\
\text { БЕЗНАЧААНО }\end{array}$ & 29 & & 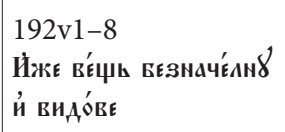 & 29 & \\
\hline
\end{tabular}




\begin{tabular}{|c|c|c|c|}
\hline & & & Tabl \\
\hline \multicolumn{3}{|c|}{ BAR MS. SL. 307} & \multirow{2}{*}{$\begin{array}{c}\text { Greek } \\
\text { Synodikon } \\
10\end{array}$} \\
\hline 7 & 8 & 9 & \\
\hline Rubrics: & $\S$ & Notes: & Rows: \\
\hline \multirow{8}{*}{$\begin{array}{l}\text { 10r7-14 } \\
\text { Гє९О́ндї̈а йЖЕ | [Ш̈ ААнЕи] }\end{array}$} & 25 & & G. $180-183$ \\
\hline & & & G. 752 \\
\hline & & & G. 753 \\
\hline & & & G. $754-755$ \\
\hline & & & G. $756-757$ \\
\hline & & & G. 758 \\
\hline & & & G. $759-760$ \\
\hline & & & G. $761-762$ \\
\hline 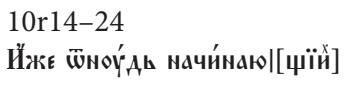 & 26 & & G. $185-189$ \\
\hline $\begin{array}{l}\text { 10r24-10v5 } \\
\text { Иैжє БАлГочи́ствоо|[ва]ти }\end{array}$ & 27 & & G. $190-192$ \\
\hline 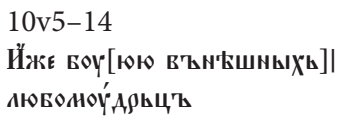 & 28 & & G. 193-197 \\
\hline 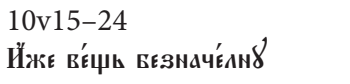 & 29 & & G. 198-202 \\
\hline
\end{tabular}




\begin{tabular}{|c|c|c|c|c|c|}
\hline \multicolumn{3}{|c|}{ Palauzov's copy } & \multicolumn{3}{|c|}{ Drinov's copy } \\
\hline 1 & 2 & 3 & 4 & 5 & 6 \\
\hline Rubrics: & $\S$ & Notes: & Rubrics: & $\S$ & Notes: \\
\hline 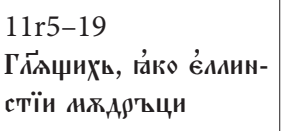 & 30 & & 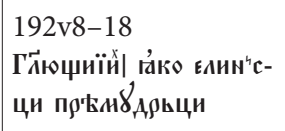 & 30 & \\
\hline 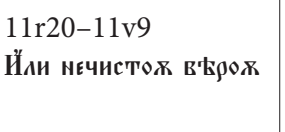 & 31 & & 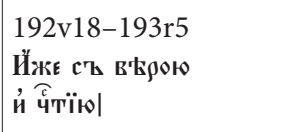 & 31 & \\
\hline 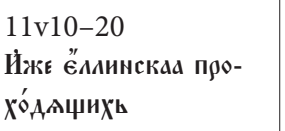 & 32 & & 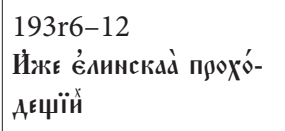 & 32 & \\
\hline 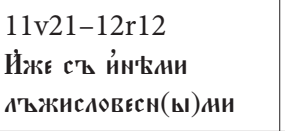 & 33 & & 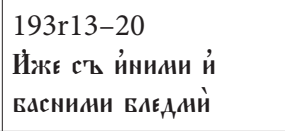 & 33 & \\
\hline 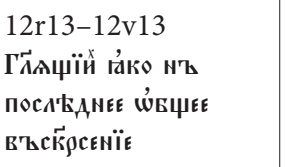 & 34 & $\begin{array}{l}\text { P 12r17-19 } \\
\text { are damaged. }\end{array}$ & 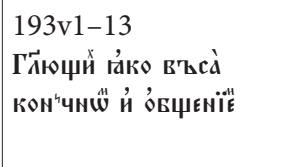 & 34 & \\
\hline \multirow[t]{6}{*}{ 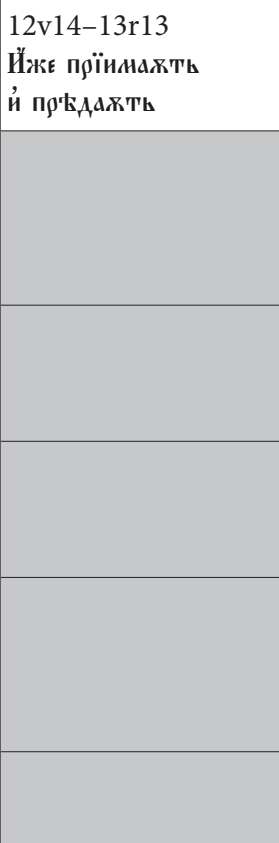 } & \multirow[t]{6}{*}{35} & & 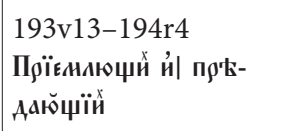 & 35 & \\
\hline & & \multirow{3}{*}{$\begin{array}{l}\text { All three exclama- } \\
\text { tions might have } \\
\text { been located on } \\
\text { the missing folios } \\
\text { between the } 18^{\text {th }} \text { and } \\
19^{\text {th }} \text { ff. in the extant } \\
\text { manuscript. See be- } \\
\text { low for the restored } \\
\text { text between } ₫ 59 \\
\text { and } ₫ 60 \text {. }\end{array}$} & 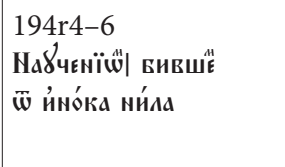 & 36 & $\begin{array}{l}\text { See } P 13 v 3-5 \\
(\$ 37) \text { and D } \\
195 \mathrm{v} 18-19 \\
(\$ 44) \text {. }\end{array}$ \\
\hline & & & 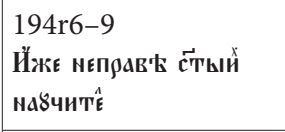 & 37 & \\
\hline & & & 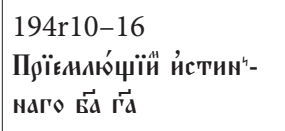 & 38 & \\
\hline & & $\begin{array}{l}\text { See P 18v7-21 } \\
(\$ 59) .\end{array}$ & 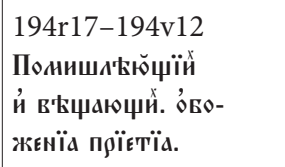 & 39 & $\begin{array}{l}\text { Corresponds to } \\
\text { P 18v7-21 ( } \$ 59) \text {, } \\
\text { whose beginning } \\
\text { is lost. }\end{array}$ \\
\hline & & $\begin{array}{l}\text { See P 19r5-20 } \\
(\$ 60) .\end{array}$ & 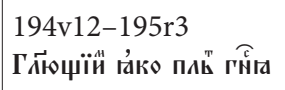 & 40 & $\begin{array}{l}\text { Corresponds to } \mathrm{P} \\
19 \mathrm{r} 5-20(\$ 60) .\end{array}$ \\
\hline
\end{tabular}


Table 1 (cont.)

\begin{tabular}{|c|c|c|c|}
\hline \multicolumn{3}{|c|}{ BAR MS. SL. 307} & \multirow{2}{*}{$\begin{array}{c}\text { Greek } \\
\text { Synodikon } \\
10\end{array}$} \\
\hline 7 & 8 & 9 & \\
\hline Rubrics: & $\$$ & Notes: & Rows: \\
\hline 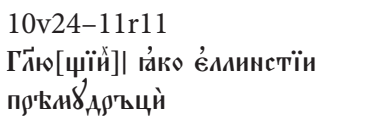 & 30 & & G. 203-208 \\
\hline 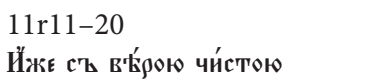 & 31 & & G. $209-213$ \\
\hline 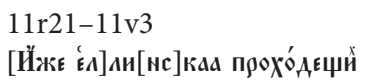 & 32 & & G. $214-218$ \\
\hline 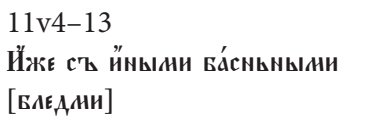 & 33 & & G. $219-224$ \\
\hline 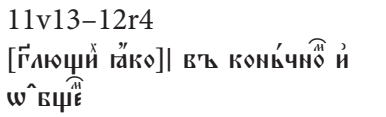 & 34 & & G. $225-233$ \\
\hline 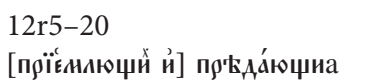 & 35 & & G. $234-242$ \\
\hline 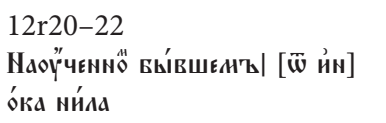 & 36 & & G. $248-249$ \\
\hline 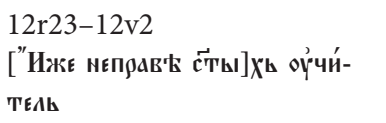 & 37 & & G. $479-481$ \\
\hline 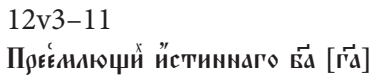 & 38 & & G. $482-487$ \\
\hline 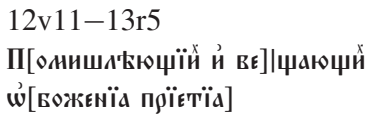 & 39 & & G. $488-497$ \\
\hline 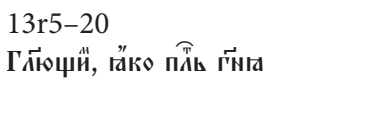 & 40 & $\begin{array}{l}\text { Discrepancies with } \mathrm{D} \text {. } \\
\text { The text coincides with the } \\
\text { Russian printed triodion. }\end{array}$ & G. $498-504$ \\
\hline
\end{tabular}




\begin{tabular}{|c|c|c|c|c|c|}
\hline \multicolumn{3}{|c|}{ Palauzov's copy } & \multicolumn{3}{|c|}{ Drinov's copy } \\
\hline 1 & 2 & 3 & 4 & 5 & 6 \\
\hline Rubrics: & $\S$ & Notes: & Rubrics: & $\$$ & Notes: \\
\hline & & $\begin{array}{l}\text { See P 19r21-19v9 } \\
(\$ 61) \text {. }\end{array}$ & 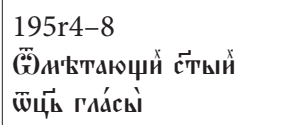 & 41 & $\begin{array}{l}\text { Corresponds to P } \\
19 \mathrm{r} 21-19 \mathrm{v} 9(\$ 61) .\end{array}$ \\
\hline & & $\begin{array}{l}\text { See P 19v10-20v2 } \\
(\$ 62) \text {. }\end{array}$ & $\begin{array}{l}\text { 195r9-195v12 } \\
\text { НєПрїЕААючй йстин'- } \\
\text { НАГО Б̆ }\end{array}$ & 42 & $\begin{array}{l}\text { Corresponds to P } \\
19 \mathrm{v} 10-20 \mathrm{v} 2(\$ 62) .\end{array}$ \\
\hline 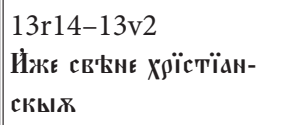 & 36 & & 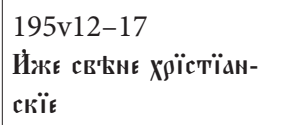 & 43 & \\
\hline 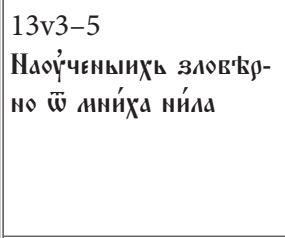 & 37 & & 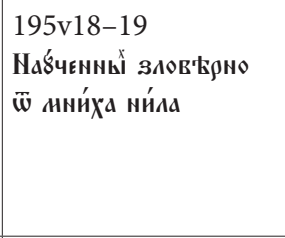 & 44 & $\begin{array}{l}\text { Repeated in D } \\
194 \mathrm{r} 4-6(\$ 36) \text {, } \\
\text { but in a different } \\
\text { redaction, which } \\
\text { coincides with P } \\
13 v 3-5(\$ 36) \text {. }\end{array}$ \\
\hline 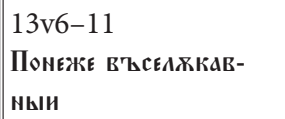 & 38 & & $\begin{array}{l}\text { 195v19-196r3 } \\
\text { ПонЕ в'МСЕ|А४КАВїи }\end{array}$ & 45 & \\
\hline $\begin{array}{l}\text { 13v12-14r4 } \\
\text { Попа в̈гшянла }\end{array}$ & 39 & & $\begin{array}{l}\text { 196r3-11 } \\
\text { По́па Ёгони́ла }\end{array}$ & 46 & \\
\hline 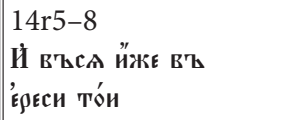 & 40 & & 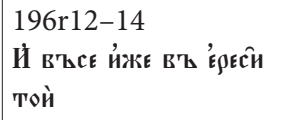 & 47 & \\
\hline $\begin{array}{l}14 \mathrm{r} 9-12 \\
\text { Пю́БАџИХ са сть } \\
\text { нияи }\end{array}$ & 41 & & 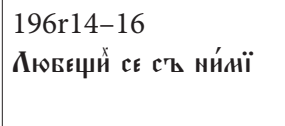 & 48 & \\
\hline 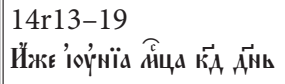 & 42 & & 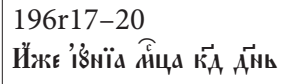 & 49 & \\
\hline $\begin{array}{l}\text { 14r20-14v3 } \\
\text { Йжє сатанд ви- } \\
\text { дия'Еи твари творца }\end{array}$ & 43 & & 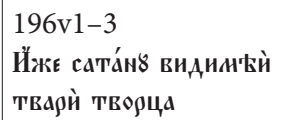 & 50 & \\
\hline 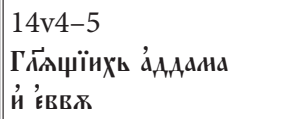 & 44 & & 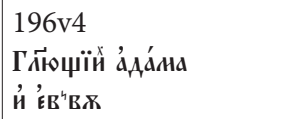 & 51 & \\
\hline 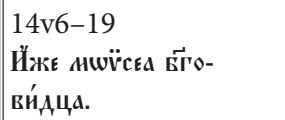 & 45 & & 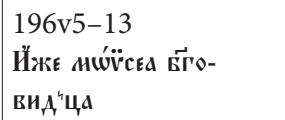 & 52 & \\
\hline
\end{tabular}




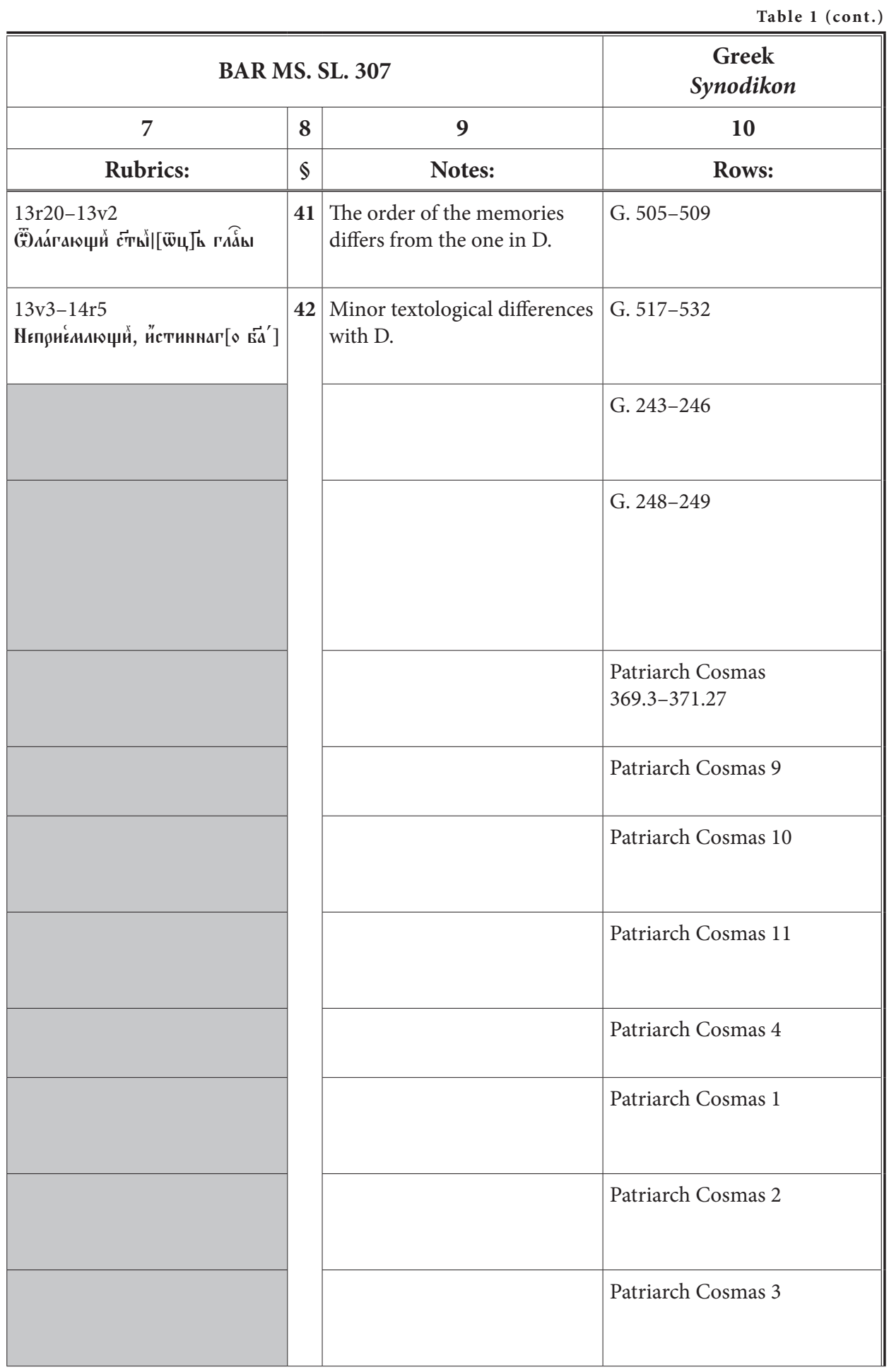




\begin{tabular}{|c|c|c|c|c|c|}
\hline \multicolumn{3}{|c|}{ Palauzov's copy } & \multicolumn{3}{|c|}{ Drinov's copy } \\
\hline 1 & 2 & 3 & 4 & 5 & 6 \\
\hline Rubrics: & $\$$ & Notes: & Rubrics: & $\S$ & Notes: \\
\hline 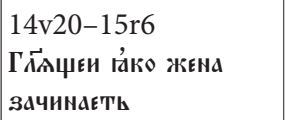 & 46 & & 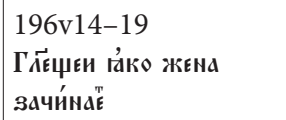 & 53 & \\
\hline 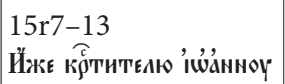 & 47 & & $\begin{array}{l}\text { 196v19-197r3 } \\
\text { Йжє кортит'єлю | 'анн8 }\end{array}$ & 54 & \\
\hline 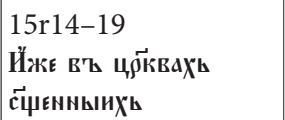 & 48 & & 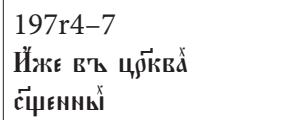 & 55 & \\
\hline 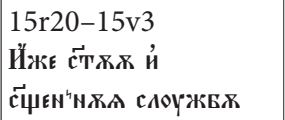 & 49 & & 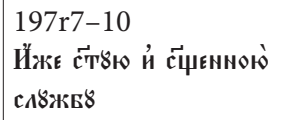 & 56 & \\
\hline 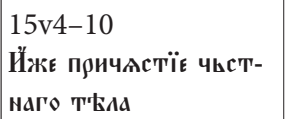 & 50 & & 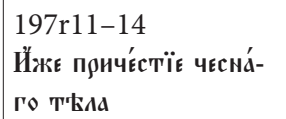 & 57 & \\
\hline 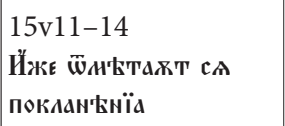 & 51 & & 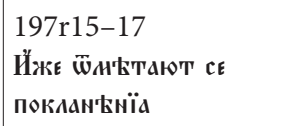 & 58 & \\
\hline 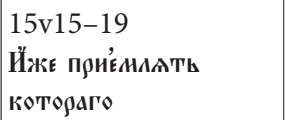 & 52 & & 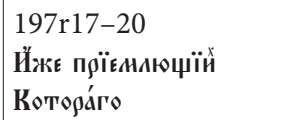 & 59 & \\
\hline $\begin{array}{l}\text { 15v20-16r3 } \\
\text { Rаси́лї̈а врача }\end{array}$ & 53 & & $\begin{array}{l}\text { 197r20-197v3 } \\
\text { Касиаїа врача }\end{array}$ & 60 & \\
\hline 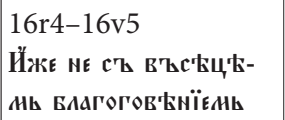 & 54 & & 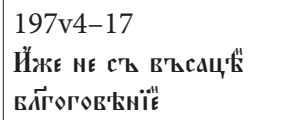 & 61 & \\
\hline 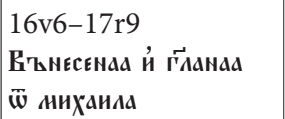 & 55 & & 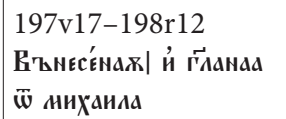 & 62 & \\
\hline 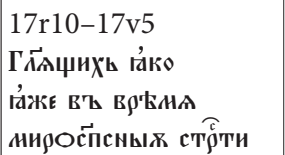 & 56 & & 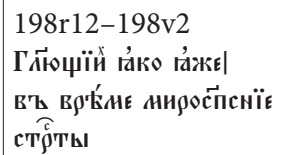 & 63 & \\
\hline 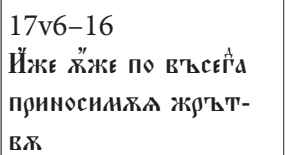 & 57 & & 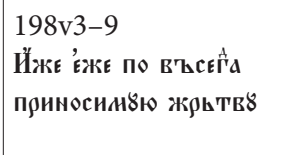 & 64 & \\
\hline
\end{tabular}




\begin{tabular}{|c|c|c|c|}
\hline \multicolumn{3}{|c|}{ BAR MS. SL. 307} & \multirow{2}{*}{$\begin{array}{c}\text { Greek } \\
\text { Synodikon } \\
10\end{array}$} \\
\hline 7 & 8 & 9 & \\
\hline Rubrics: & $\S$ & Notes: & Rows: \\
\hline & & & Patriarch Cosmas 4 \\
\hline & & & Patriarch Cosmas 5 \\
\hline & & & Patriarch Cosmas 6 \\
\hline & & & Patriarch Cosmas 7 \\
\hline & & & Patriarch Cosmas 8 \\
\hline & & & Patriarch Cosmas 12 \\
\hline & & & G. $395-403$ \\
\hline & & & G. $424-434$ \\
\hline & & & G. $435-443$ \\
\hline & & & G. $444-448$ \\
\hline
\end{tabular}




\begin{tabular}{|c|c|c|c|c|c|}
\hline \multicolumn{3}{|c|}{ Palauzov's copy } & \multicolumn{3}{|c|}{ Drinov's copy } \\
\hline 1 & 2 & 3 & 4 & 5 & 6 \\
\hline Rubrics: & $\S$ & Notes: & Rubrics: & $\$$ & Notes: \\
\hline 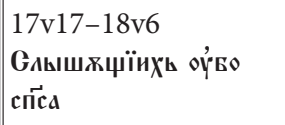 & 58 & & 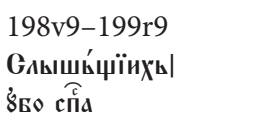 & 65 & \\
\hline 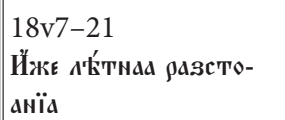 & 59 & $\begin{array}{l}\text { The end, correspond- } \\
\text { ing to D 199r18-21, } \\
\text { is missing. }\end{array}$ & 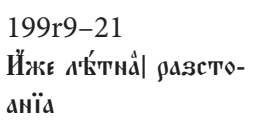 & 66 & \\
\hline 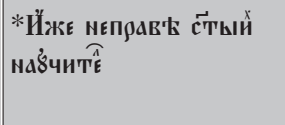 & & $\begin{array}{l}\text { The text is restored } \\
\text { according to D } \\
194 \mathrm{r} 6-9(\$ 37) \text {. }\end{array}$ & & & \\
\hline 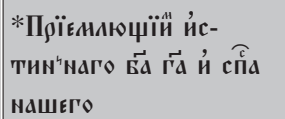 & & $\begin{array}{l}\text { The text is restored } \\
\text { according to D } \\
194 \mathrm{r} 10-16(\$ 38) \text {. }\end{array}$ & & & \\
\hline 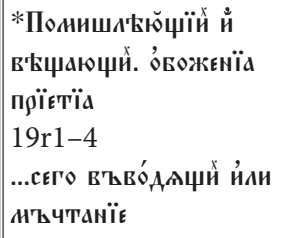 & & $\begin{array}{l}\text { The beginning is } \\
\text { restored according } \\
\text { to D } 194 \mathrm{r} 17-194 \mathrm{v} 9 \text {, } \\
\text { the end in P } 19 \mathrm{r} 1-4 \\
\text { coincides with D } \\
194 \mathrm{v} 9-13(\$ 39) \text {. }\end{array}$ & & & \\
\hline 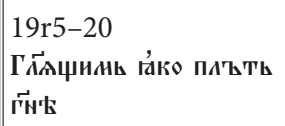 & 60 & $\begin{array}{l}\text { Coincides with D } \\
\text { 194v12-195r3 ( } \$ 40) .\end{array}$ & & & \\
\hline 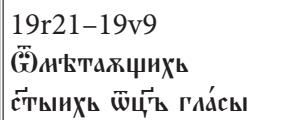 & 61 & $\begin{array}{l}\text { Coincides with D } \\
\text { 195r4-8 (\$ } 41) \text {. }\end{array}$ & & & \\
\hline 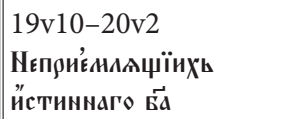 & 62 & $\begin{array}{l}\text { Coincides with D } \\
\text { 195r9-195v12 (\$ 42). }\end{array}$ & & & \\
\hline 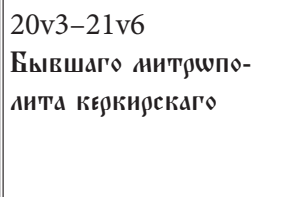 & 63 & & $\begin{array}{l}\text { 199v1-20 } \\
\text { Бывшаго нитршпо- } \\
\text { Аита керкирскаго }\end{array}$ & 67 & $\begin{array}{l}\text { The end of the text } \\
\text { is missing due to } \\
\text { loss of folios. The } \\
\text { next text coincides } \\
\text { with P } 24 \text { r } 12 \text { sq. }\end{array}$ \\
\hline
\end{tabular}


Table 1 (cont.)

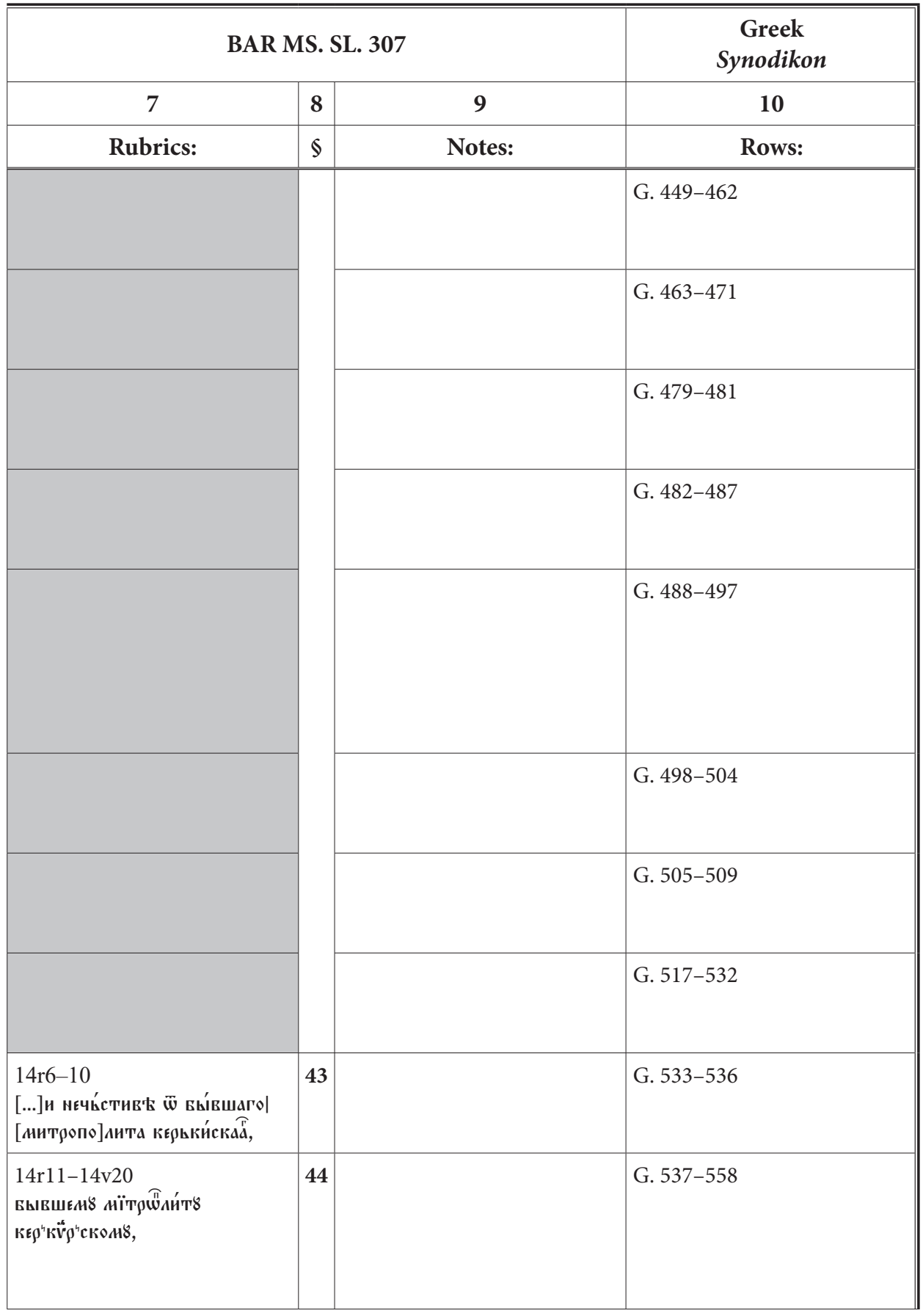




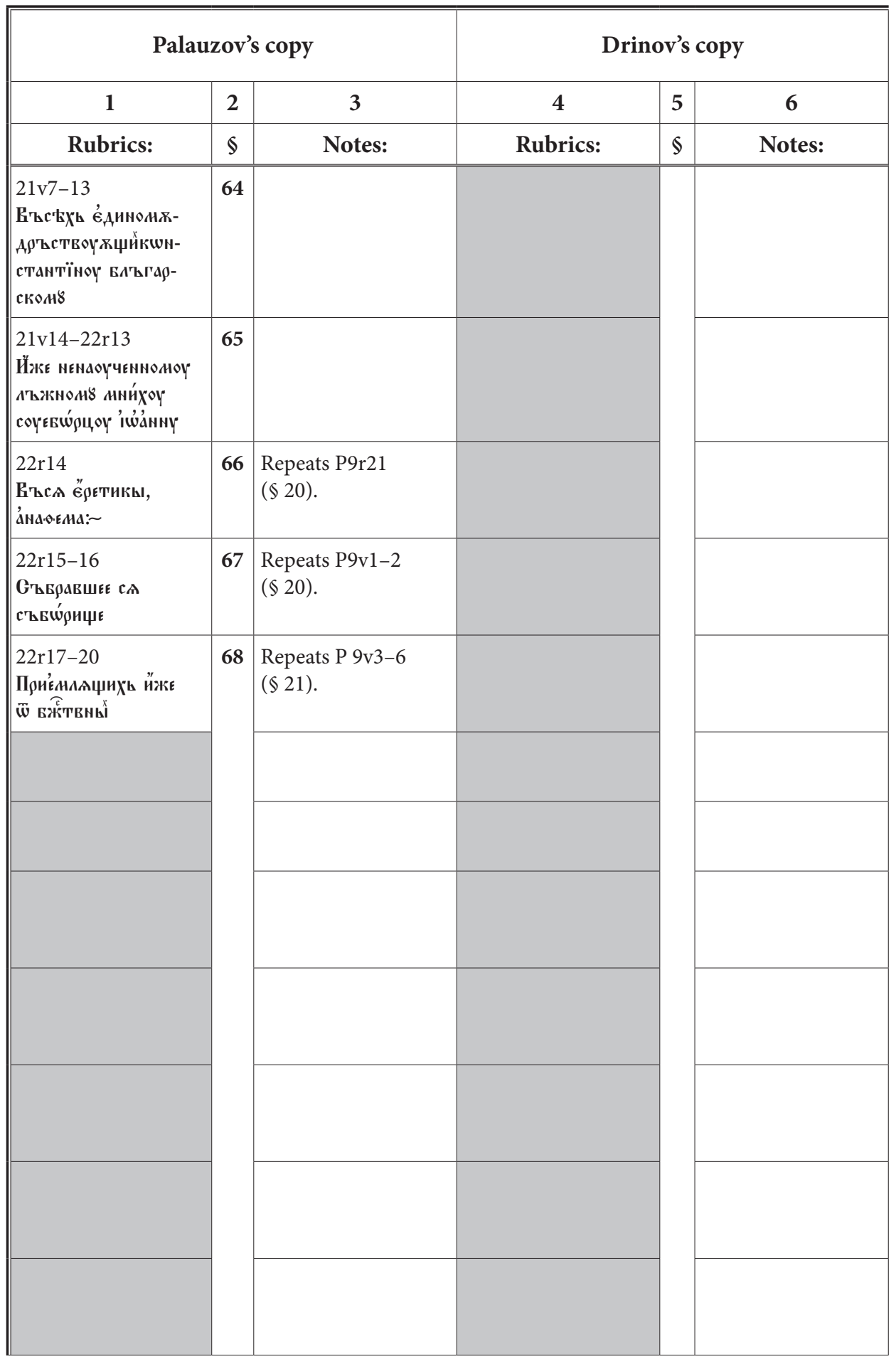


Table 1 (cont.)

\begin{tabular}{|c|c|c|c|}
\hline \multicolumn{3}{|c|}{ BAR MS. SL. 307} & \multirow{2}{*}{$\begin{array}{c}\text { Greek } \\
\text { Synodikon } \\
10\end{array}$} \\
\hline 7 & 8 & 9 & \\
\hline Rubrics: & $\S$ & Notes: & Rows: \\
\hline 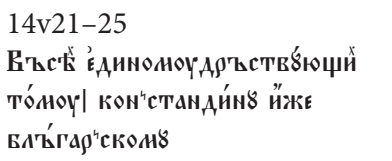 & 45 & & G. $558-561$ \\
\hline \multirow[t]{2}{*}{ 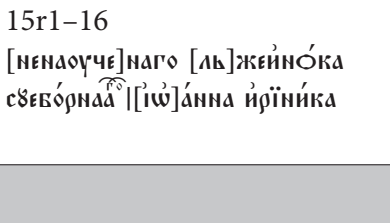 } & 46 & & G. $562-571$ \\
\hline & & & G. 752 \\
\hline 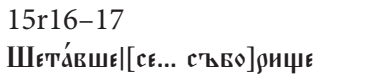 & 47 & See P 9v1-2 (\$20). & G. 753 \\
\hline 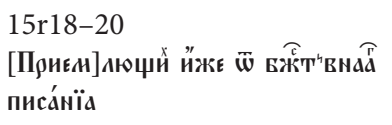 & 48 & See P 9v3-6 (\$21). & G. $754-755$ \\
\hline 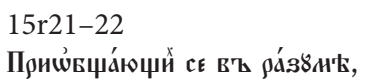 & 49 & See P 9v7-9 (\$22). & G. $756-757$ \\
\hline 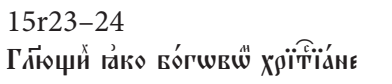 & 50 & See P 9v10-12 (\$23). & G. 758 \\
\hline 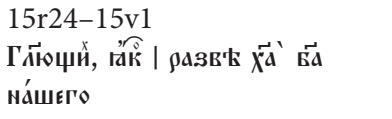 & 51 & See P 9v13-15 (\$24). & G. $759-760$ \\
\hline 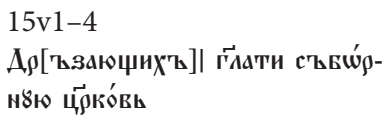 & 52 & See P 9v16-20 (\$25). & G. $761-762$ \\
\hline 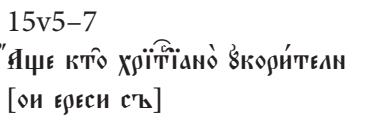 & 53 & & G. $763-764$ \\
\hline 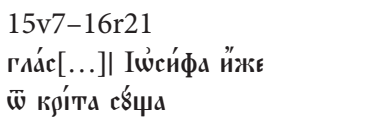 & 54 & 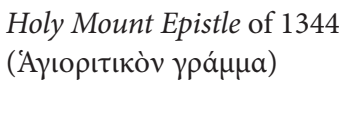 & $\begin{array}{l}\text { A. Rigo, L'Assemblea gene- } \\
\text { rale..., p. } 505 \text {, fig. } 31-56 .\end{array}$ \\
\hline 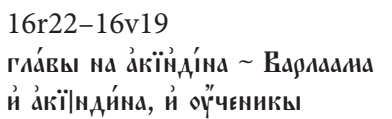 & 55 & Cf. P 27r10-27v6 (\$176). & G. 573-584 \\
\hline
\end{tabular}




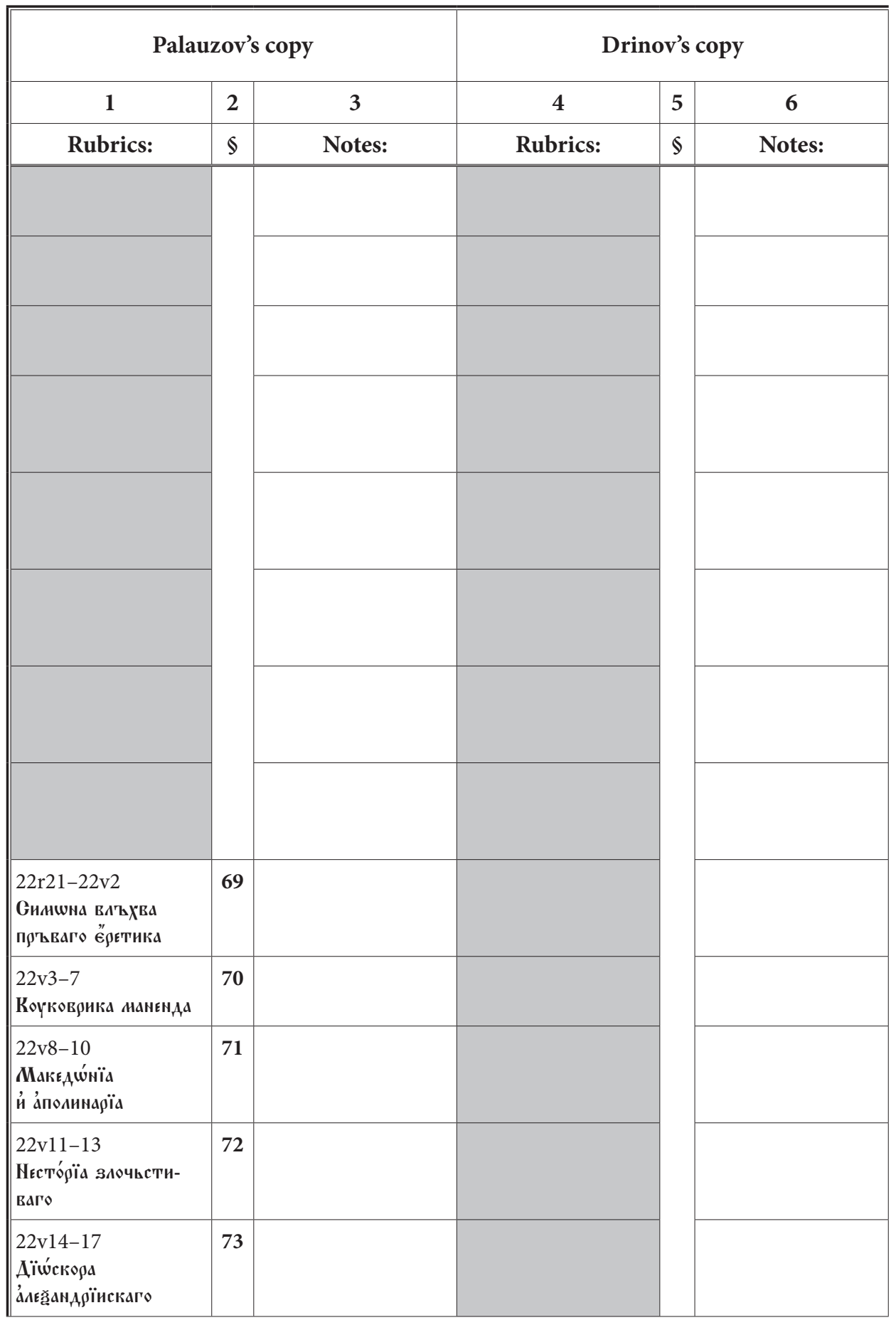


Table 1 (cont.)

\begin{tabular}{|c|c|c|c|}
\hline \multicolumn{3}{|c|}{ BAR MS. SL. 307} & \multirow{2}{*}{$\begin{array}{c}\text { Greek } \\
\text { Synodikon } \\
10\end{array}$} \\
\hline 7 & 8 & 9 & \\
\hline Rubrics: & $\S$ & Notes: & Rows: \\
\hline 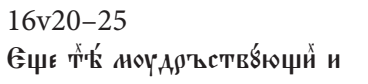 & 56 & $\begin{array}{l}\text { No ending due to loss of } \\
\text { folios. }\end{array}$ & G. $585-587$ \\
\hline 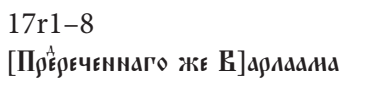 & 57 & $\begin{array}{l}\text { Memory to Andronicus III } \\
\text { Palaeologus. }\end{array}$ & G. $687-691$ \\
\hline 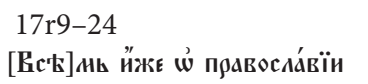 & 58 & & G. $714-732$ \\
\hline 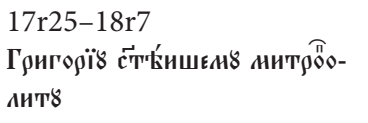 & 59 & & G. $692-709$ \\
\hline 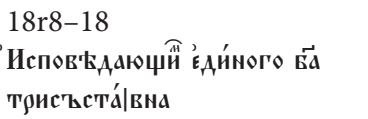 & 60 & & G. $724-729$ \\
\hline 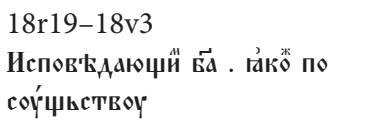 & 61 & & G. $730-734$ \\
\hline 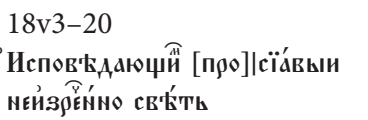 & 62 & & G. $735-743$ \\
\hline \multirow[t]{5}{*}{ 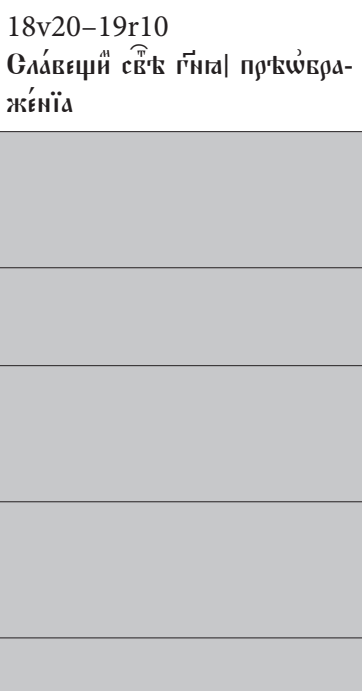 } & 63 & & G. $744-751$ \\
\hline & & & G. $295.74-75$ \\
\hline & & & G. $295.76-78$ \\
\hline & & & G. $296.79-81$ \\
\hline & & & G. $296.79-82$ \\
\hline
\end{tabular}




\begin{tabular}{|c|c|c|c|c|c|}
\hline \multicolumn{3}{|c|}{ Palauzov's copy } & \multicolumn{3}{|c|}{ Drinov's copy } \\
\hline 1 & 2 & 3 & 4 & 5 & 6 \\
\hline Rubrics: & $\S$ & Notes: & Rubrics: & $\$$ & Notes: \\
\hline \begin{tabular}{|l}
$22 \mathrm{v} 18-21$ \\
ООИГЕна БєзоүнанаГО
\end{tabular} & 74 & & & & \\
\hline 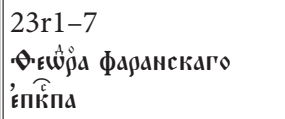 & 75 & & & & \\
\hline $\begin{array}{l}\text { 23r8-10 } \\
\text { Па́вАа саноосадскаго. }\end{array}$ & 76 & & & & \\
\hline 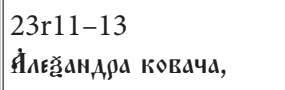 & 77 & & & & \\
\hline 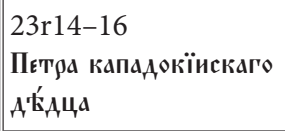 & 78 & & & & \\
\hline 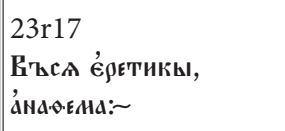 & 79 & $\begin{array}{l}\text { Compare P 22r14 } \\
(\$ 66) .\end{array}$ & & & \\
\hline 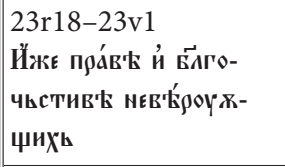 & 80 & & & & \\
\hline 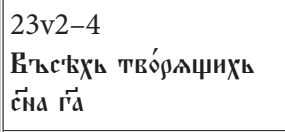 & 81 & & & & \\
\hline $\begin{array}{l}23 \mathrm{v} 5-7 \\
\text { ӤЖЕ нЕ Йспов'ВАОГЕтТ }\end{array}$ & 82 & & & & \\
\hline 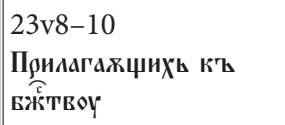 & 83 & & & & \\
\hline 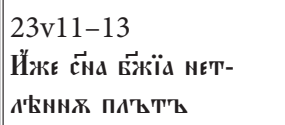 & 84 & & & & \\
\hline 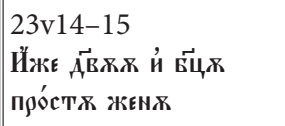 & 85 & & & & \\
\hline
\end{tabular}




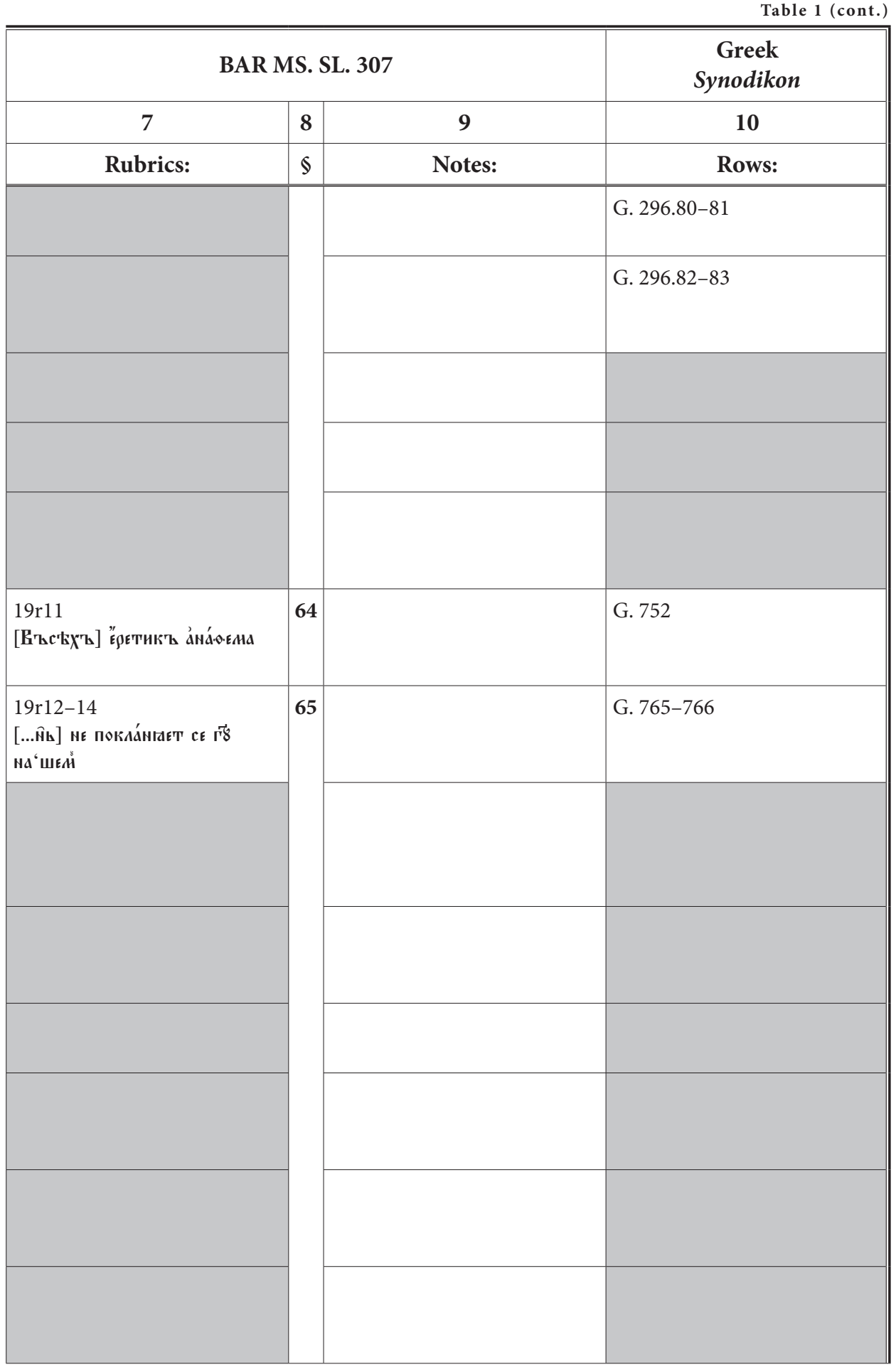




\begin{tabular}{|c|c|c|c|c|c|}
\hline \multicolumn{3}{|c|}{ Palauzov's copy } & \multicolumn{3}{|c|}{ Drinov's copy } \\
\hline 1 & 2 & 3 & 4 & 5 & 6 \\
\hline Rubrics: & $\S$ & Notes: & Rubrics: & $\S$ & Notes: \\
\hline 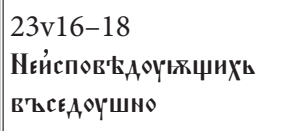 & 86 & & & & \\
\hline 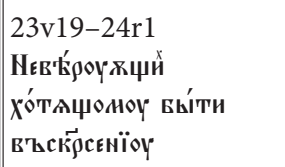 & 87 & & & & \\
\hline 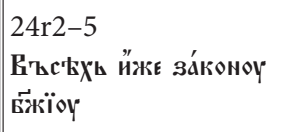 & 88 & & & & \\
\hline $\begin{array}{l}24 \mathrm{r6-9} \\
\text { Н̈жє ш̄ каковы АнБо } \\
\text { Е९єси }\end{array}$ & 89 & & & & \\
\hline 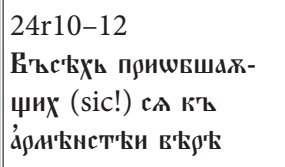 & 90 & & & & \\
\hline 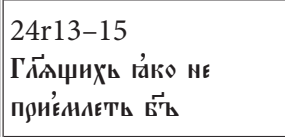 & 91 & & 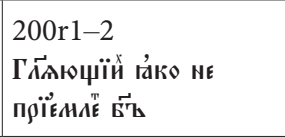 & 68 & \\
\hline 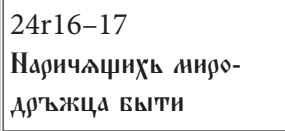 & 92 & & 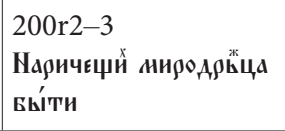 & 69 & \\
\hline 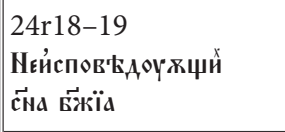 & 93 & & 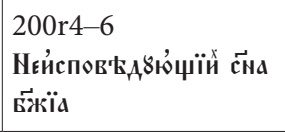 & 70 & \\
\hline 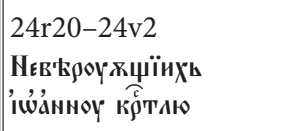 & 94 & & 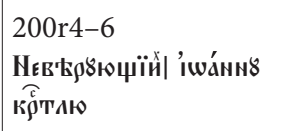 & 71 & \\
\hline 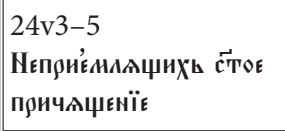 & 95 & & 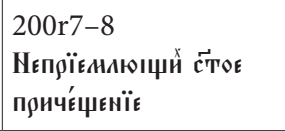 & 72 & \\
\hline 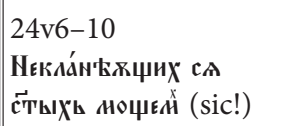 & 96 & & 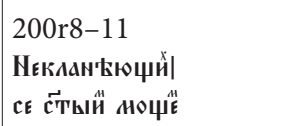 & 73 & \\
\hline
\end{tabular}




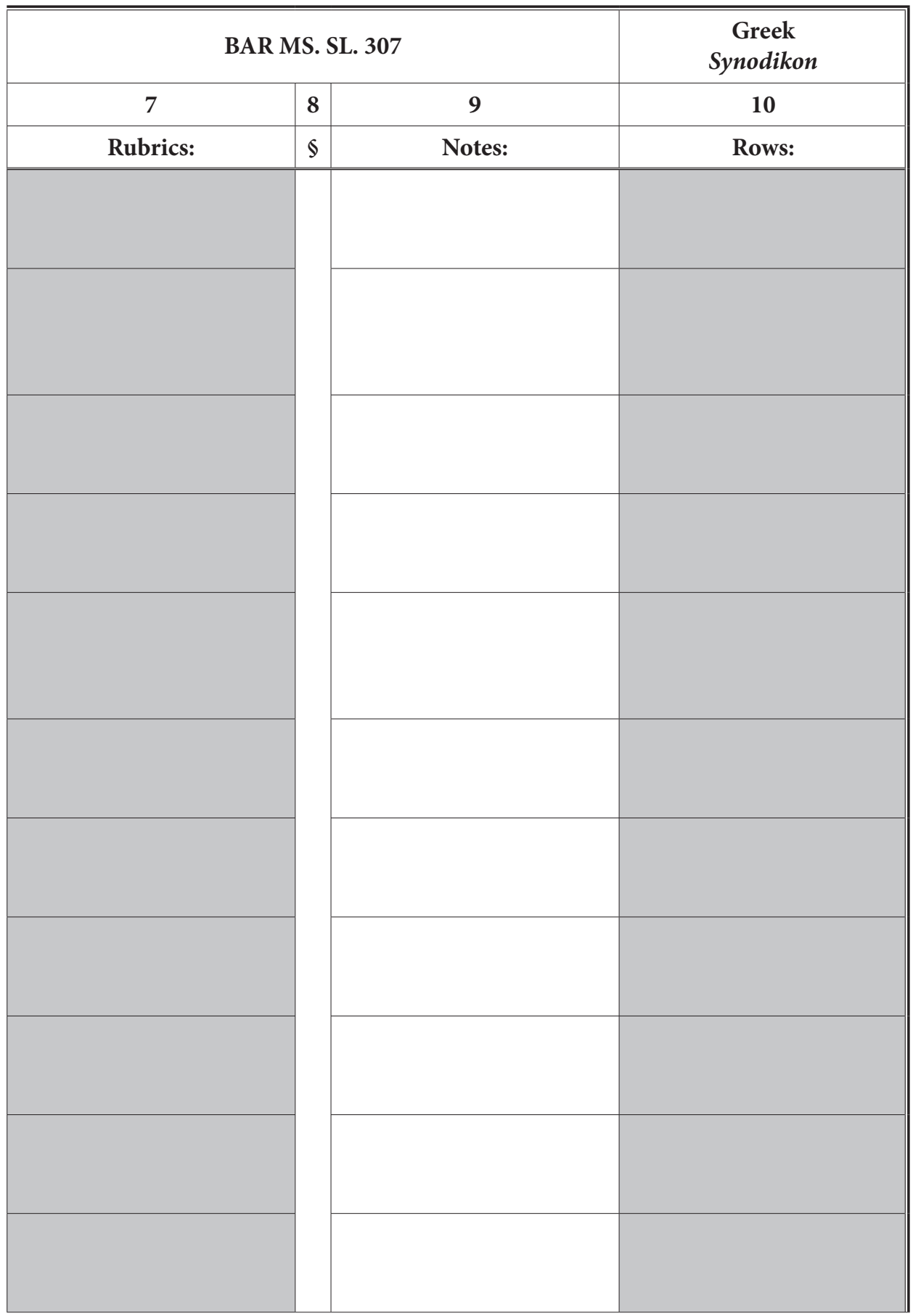




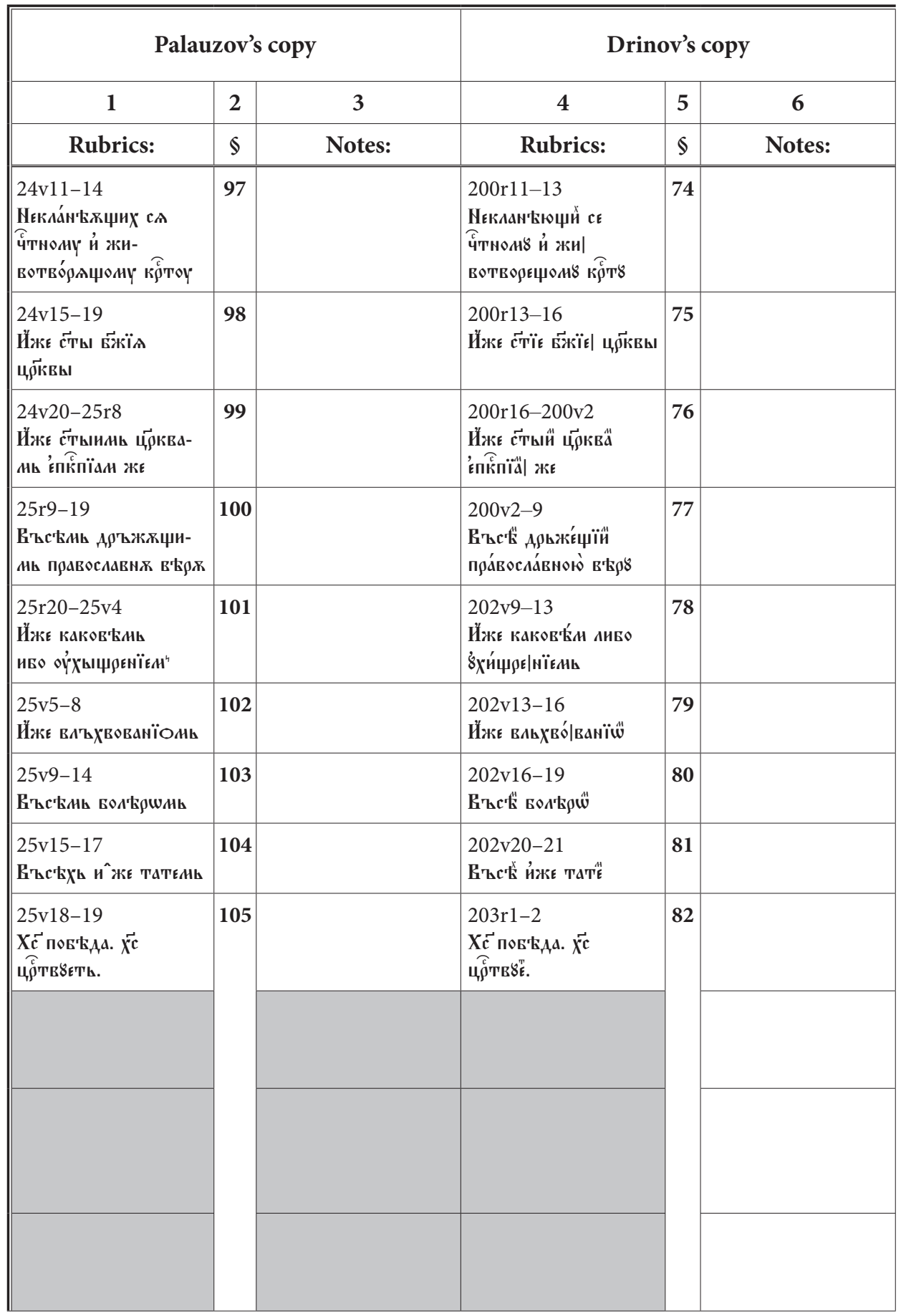


Table 1 (cont.)

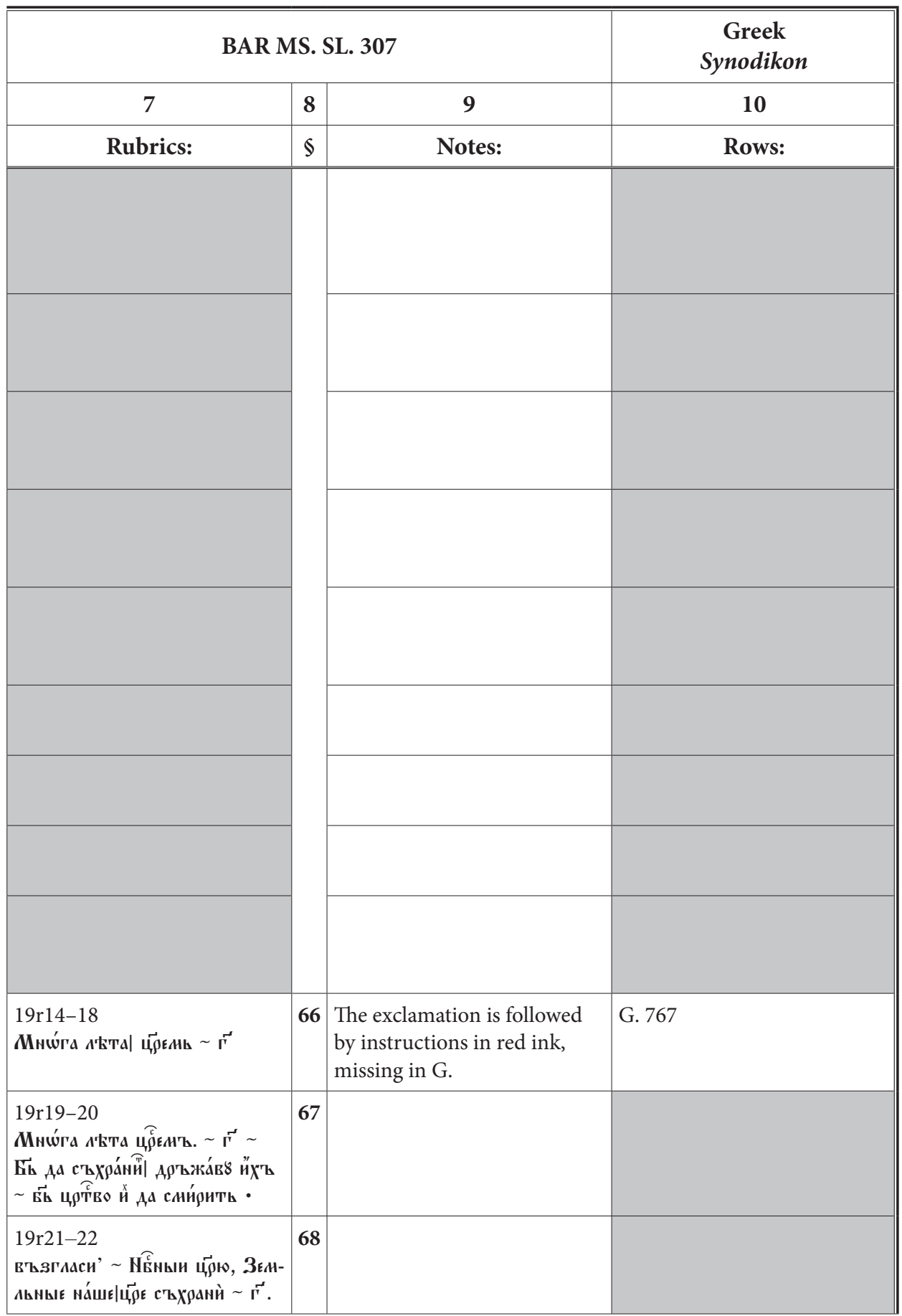




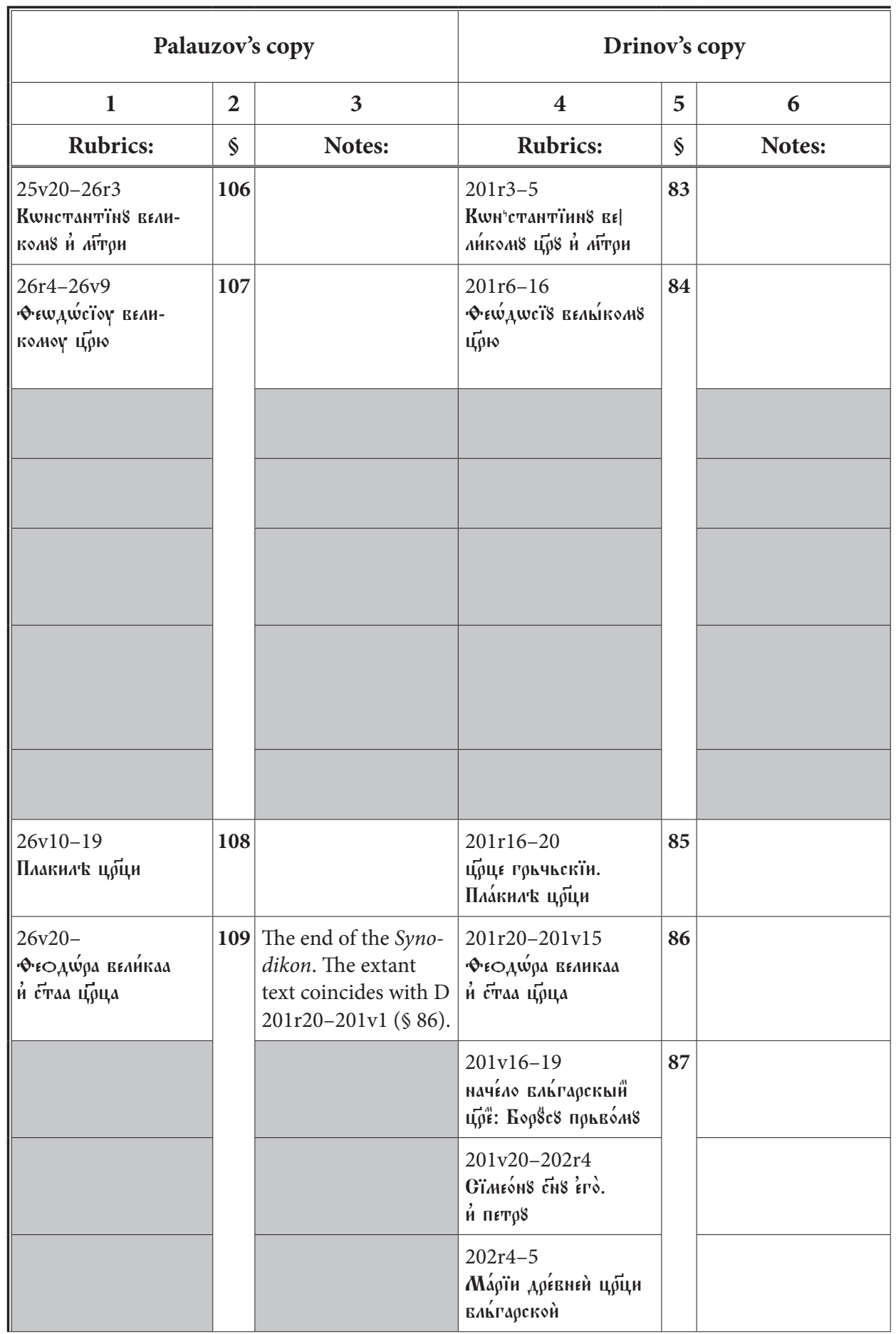


Table 1 (cont.)

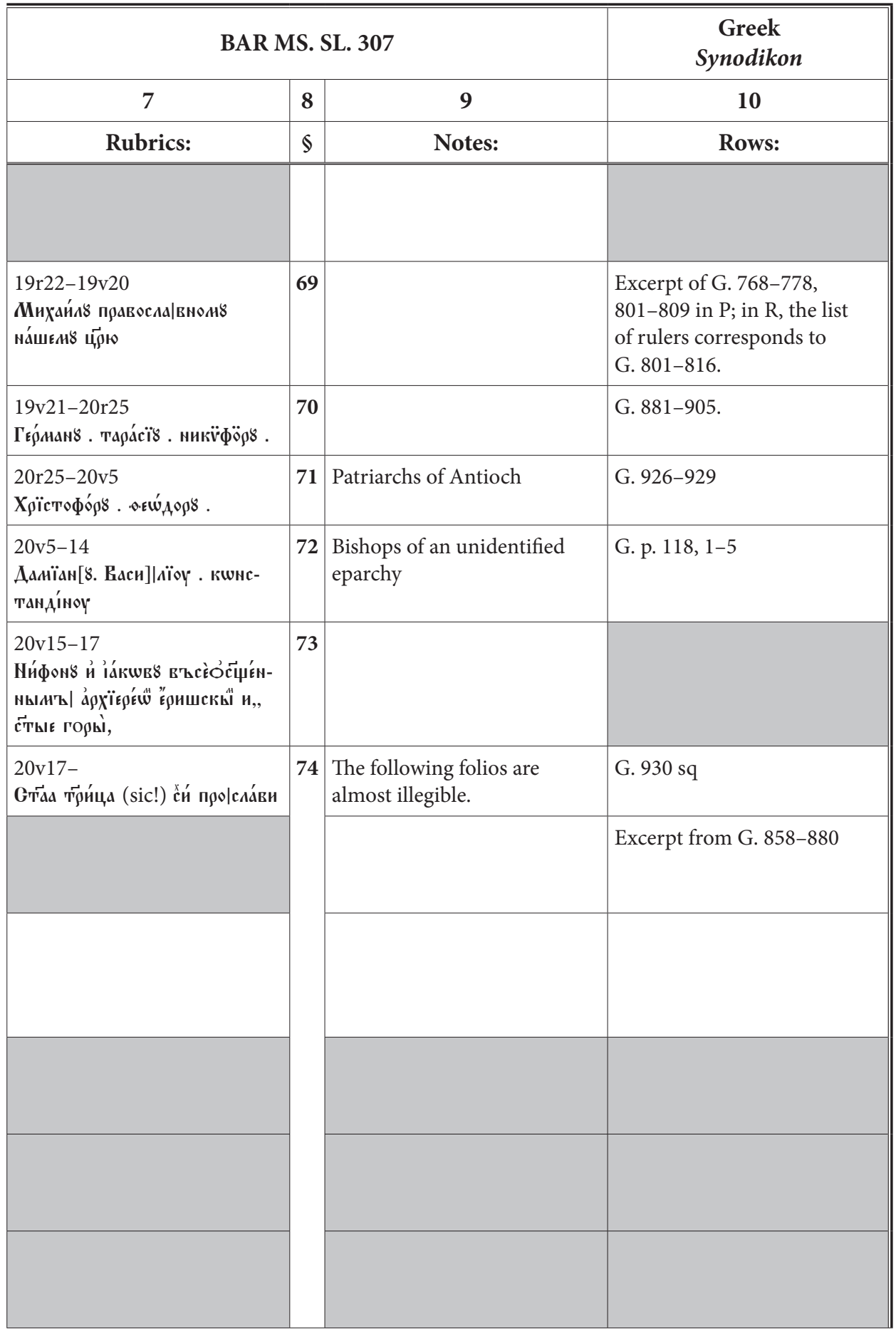




\begin{tabular}{|c|c|c|c|c|c|}
\hline \multicolumn{3}{|c|}{ Palauzov's copy } & \multicolumn{3}{|c|}{ Drinov's copy } \\
\hline 1 & 2 & 3 & 4 & 5 & 6 \\
\hline Rubrics: & $\$$ & Notes: & Rubrics: & $\$$ & Notes: \\
\hline & & & 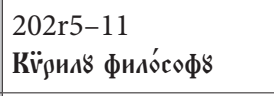 & 88 & \\
\hline & & & 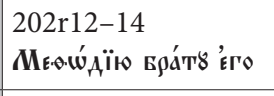 & 89 & \\
\hline & & & 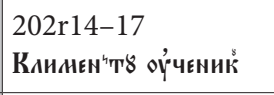 & 90 & \\
\hline & & & 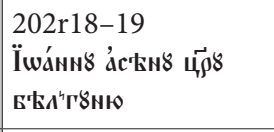 & 91 & \\
\hline & & & 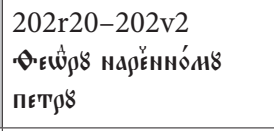 & 92 & \\
\hline $28 \mathrm{r} 1-29 \mathrm{r} 4$ & & $\begin{array}{l}\text { The beginning of } \\
\text { the story about } \\
\text { the Synod of } 1211 \text {, } \\
\text { corresponding to D } \\
202 \mathrm{v} 3-14 \text {, is miss- } \\
\text { ing. P } 28 \mathrm{r} 1-8 \mathrm{v} 21 \\
\text { coincides with D } \\
202 \mathrm{v} 14-203 \mathrm{r} 20 \text {. }\end{array}$ & 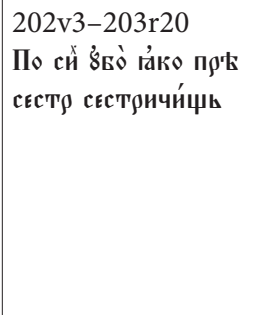 & 93 & $\begin{array}{l}\text { The end of the story } \\
\text { about the Synod, } \\
\text { corresponding to P } \\
29 \mathrm{r}-32 \mathrm{v} \text {, is missing. }\end{array}$ \\
\hline $\begin{array}{l}29 \mathrm{r} 4-16 \\
\text { Й ПО } \mathfrak{c E A เ ~ П О В Е А ' ~}\end{array}$ & 110 & & & & \\
\hline 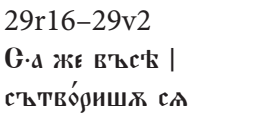 & & & & & \\
\hline 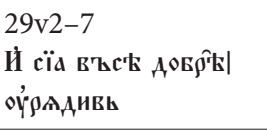 & & & & & \\
\hline $\begin{array}{l}29 \mathrm{v} 8-17 \\
\text { Т९ъкААтаго Бого- } \\
\text { АнАа }\end{array}$ & 111 & & & & \\
\hline 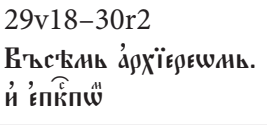 & 112 & & & & \\
\hline 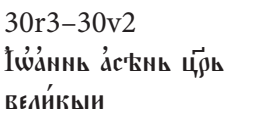 & 113 & & & & \\
\hline
\end{tabular}


Table 1 (cont.)

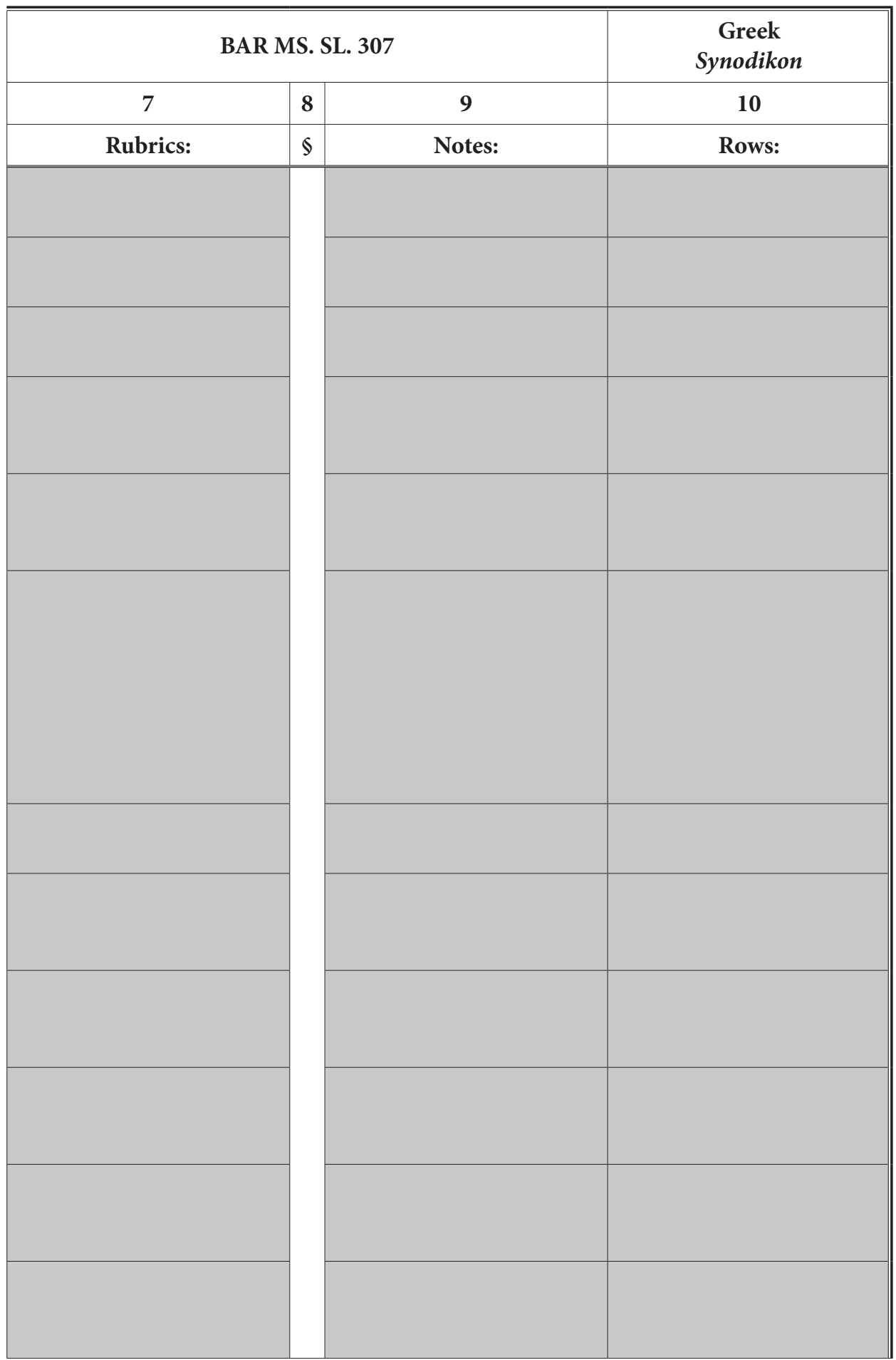




\begin{tabular}{|c|c|c|c|c|c|}
\hline \multicolumn{3}{|c|}{ Palauzov's copy } & \multicolumn{3}{|c|}{ Drinov's copy } \\
\hline 1 & 2 & 3 & 4 & 5 & 6 \\
\hline Rubrics: & $\$$ & Notes: & Rubrics: & $\S$ & Notes: \\
\hline 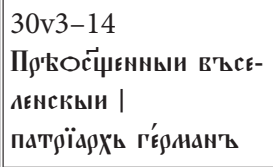 & & & & & \\
\hline 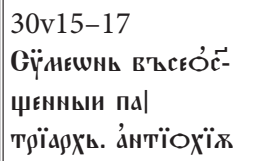 & & & & & \\
\hline 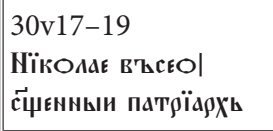 & & & & & \\
\hline 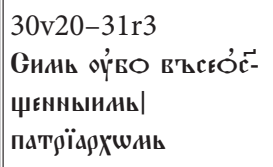 & & & & & \\
\hline 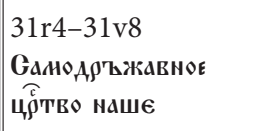 & & & & & \\
\hline 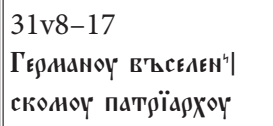 & 113 & & & & \\
\hline 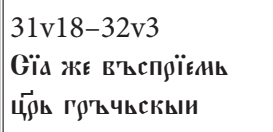 & & & & & \\
\hline 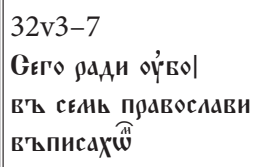 & & & & & \\
\hline 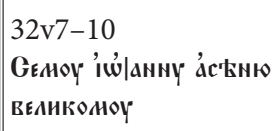 & & & & & \\
\hline $\begin{array}{l}32 \mathrm{v} 11-15 \\
\text { Калинаноү БӓговТ } \rho- \\
\text { ноноу ц्рю. }\end{array}$ & 114 & & & & \\
\hline 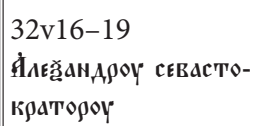 & 115 & & & & \\
\hline
\end{tabular}


Table 1 (cont.)

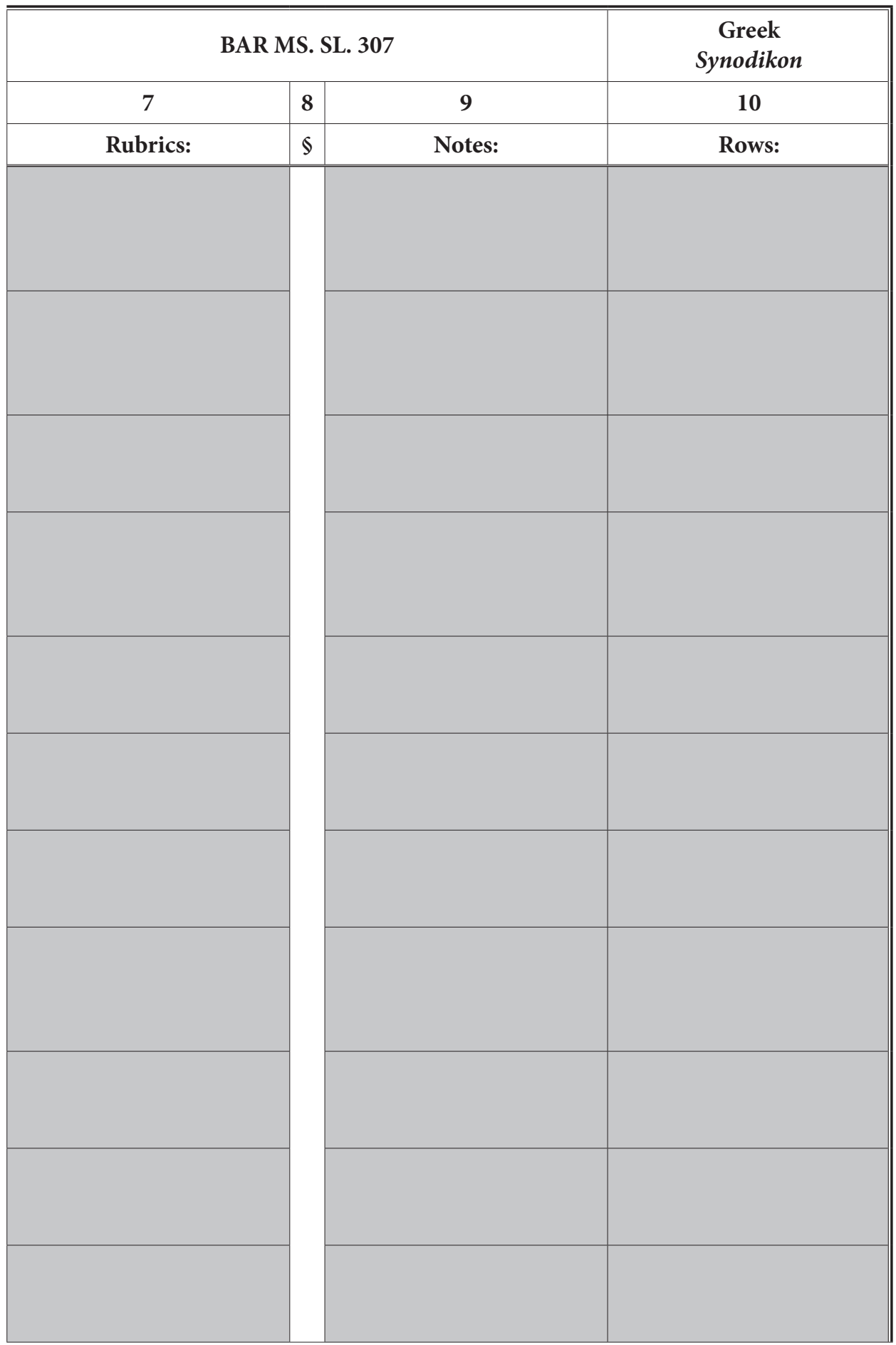




\begin{tabular}{|c|c|c|c|c|c|}
\hline \multicolumn{3}{|c|}{ Palauzov's copy } & \multicolumn{3}{|c|}{ Drinov's copy } \\
\hline 1 & 2 & 3 & 4 & 5 & 6 \\
\hline Rubrics: & $\S$ & Notes: & Rubrics: & $\S$ & Notes: \\
\hline \multirow[t]{11}{*}{$\begin{array}{l}\text { 32v20- } \\
\text { Кшнстантїну Б̈лго- } \\
\text { Чьстивон(оу) }\end{array}$} & 116 & $\begin{array}{l}\text { The end of the glori- } \\
\text { fication is missing, as } \\
\text { are the glories to the } \\
\text { next Bulgarian tsars } \\
\text { partially preserved } \\
\text { in D 203r20-203v19 } \\
\text { (\$94). }\end{array}$ & & & \\
\hline & & & 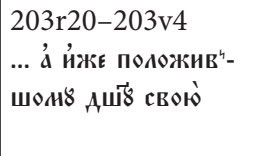 & 94 & $\begin{array}{l}\text { It seems to be the } \\
\text { end of the glory to } \\
\text { Constantine Asen } \\
(1258-1277) \text {. }\end{array}$ \\
\hline & & & 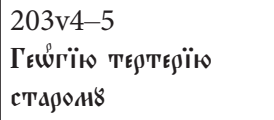 & 95 & \\
\hline & & & 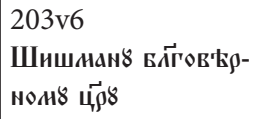 & 96 & \\
\hline & & & 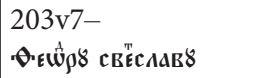 & 97 & \\
\hline & & & 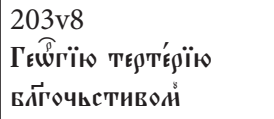 & 98 & \\
\hline & & & $\begin{array}{l}\text { 203v9-10 } \\
\text { Отра́цинйрళ Аєспо́т૪. }\end{array}$ & 99 & \\
\hline & & & $\begin{array}{l}203 \mathrm{v} 10-11 \\
\text { МиханАв БйГОчьстти- } \\
\text { вон8 }\end{array}$ & 100 & \\
\hline & & & 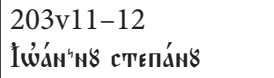 & 101 & \\
\hline & & & 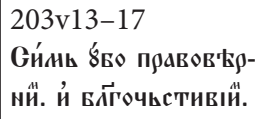 & 102 & \\
\hline & & & 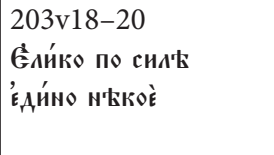 & 103 & $\begin{array}{l}\text { The end of the glo- } \\
\text { rification is missing, } \\
\text { as is the end of the } \\
\text { Synodikon, in D. }\end{array}$ \\
\hline
\end{tabular}


Table 1 (cont.)

\begin{tabular}{|c|c|c|c|}
\hline \hline \multicolumn{3}{|c|}{ BAR MS. SL. 307 } & Greek \\
Synodikon
\end{tabular}




\begin{tabular}{|c|c|c|c|c|c|}
\hline \multicolumn{3}{|c|}{ Palauzov's copy } & \multicolumn{3}{|c|}{ Drinov's copy } \\
\hline 1 & 2 & 3 & 4 & 5 & 6 \\
\hline Rubrics: & $\$$ & Notes: & Rubrics: & $\$$ & Notes: \\
\hline $\begin{array}{l}\text { 34r1-5 } \\
\text { GАЕн' НовТИ БАГО- } \\
\text { ЧьстИвТИ }\end{array}$ & 117 & & & & \\
\hline 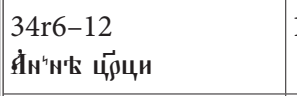 & 118 & & & & \\
\hline 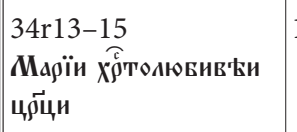 & 119 & & & & \\
\hline 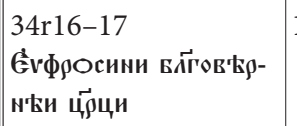 & 120 & & & & \\
\hline 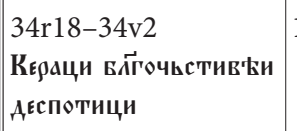 & 121 & & & & \\
\hline 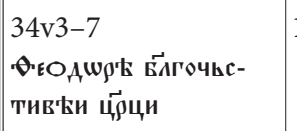 & 122 & & & & \\
\hline 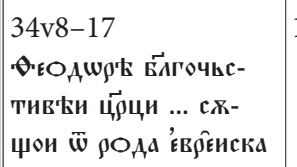 & 123 & & & & \\
\hline 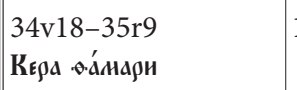 & 124 & & & & \\
\hline $\begin{array}{l}35 \mathrm{r} 10-11 \\
\text { Гж⿱乛и Аєсислав' й гж⿱乛龰и } \\
\text { василиси }\end{array}$ & 125 & & & & \\
\hline $\begin{array}{l}\text { 35r12-14 } \\
\text { Кїра марїи }\end{array}$ & 126 & & & & \\
\hline 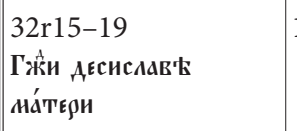 & 127 & & & & \\
\hline $\begin{array}{l}\text { 35r20-35v3 } \\
\text { К'єраци. А'ъщєри }\end{array}$ & 128 & & & & \\
\hline 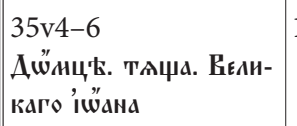 & 129 & & & & \\
\hline
\end{tabular}




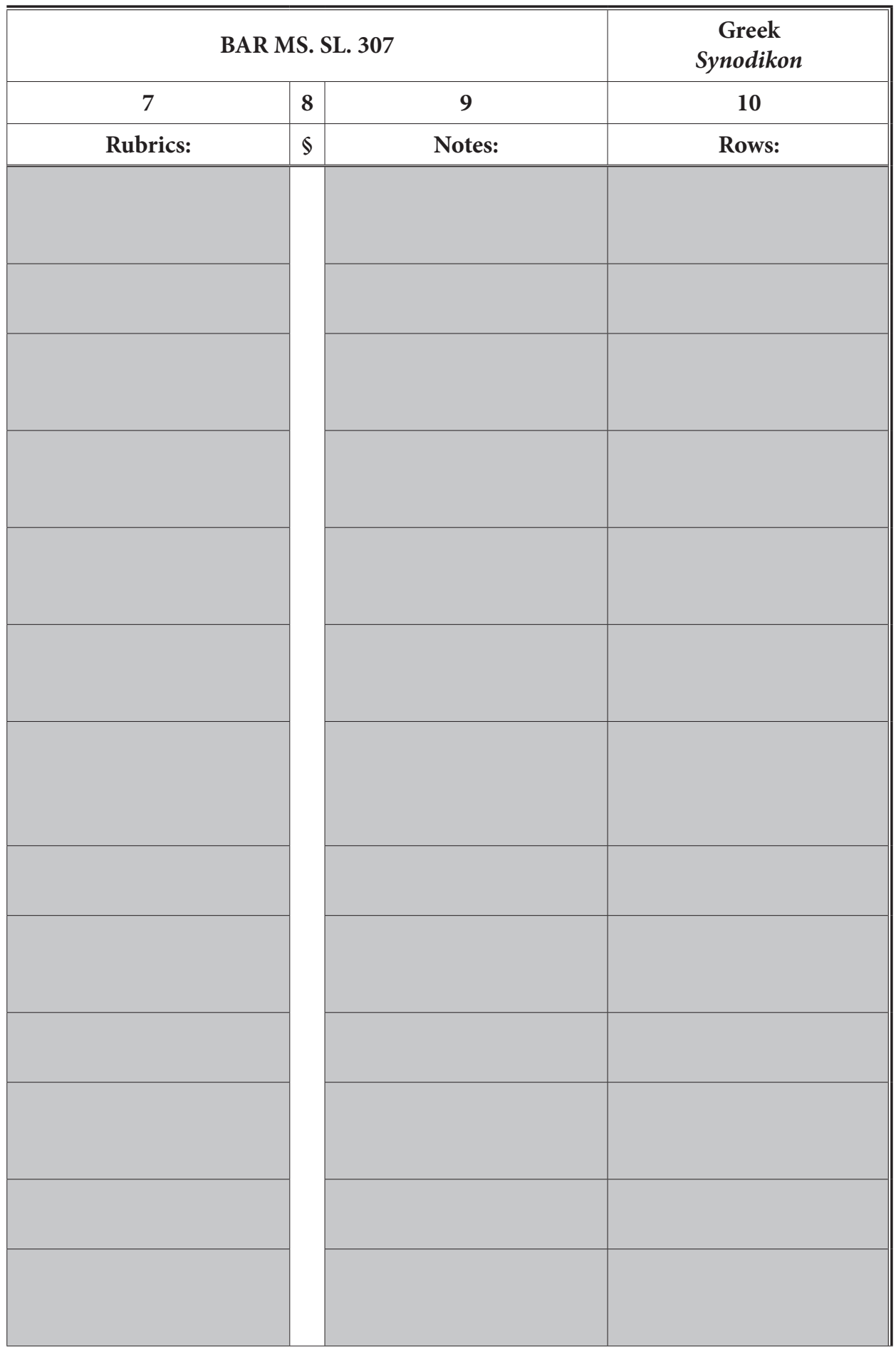




\begin{tabular}{|c|c|c|c|c|c|}
\hline \multicolumn{3}{|c|}{ Palauzov's copy } & \multicolumn{3}{|c|}{ Drinov's copy } \\
\hline 1 & 2 & 3 & 4 & 5 & 6 \\
\hline Rubrics: & $\$$ & Notes: & Rubrics: & $\S$ & Notes: \\
\hline 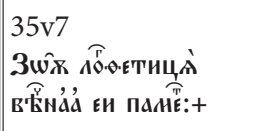 & 130 & $\begin{array}{l}\text { The lower half of the } \\
\text { folio is empty. }\end{array}$ & & & \\
\hline $\begin{array}{l}\text { 33r1-3 } \\
\text { Мшнахоу сїлвестря8. }\end{array}$ & 131 & & & & \\
\hline 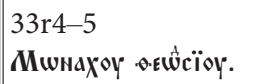 & 132 & & & & \\
\hline $\begin{array}{l}33 \mathrm{r6} \\
\text { ПротокелїОтиноу } \\
\text { пюданкоу }\end{array}$ & 133 & & & & \\
\hline $\begin{array}{l}\text { 33r7-9 } \\
\text { Протокєиї̈тиноу } \\
\text { прїгазд'Е }\end{array}$ & 134 & & & & \\
\hline $\begin{array}{l}33 \mathrm{r} 10 \\
\text { ЦанЕьАКоу }\end{array}$ & 135 & & & & \\
\hline $\begin{array}{l}\text { 33r11-13 } \\
\text { ВЕли́коду воєвШАС } \\
\text { КШнстантїноу. }\end{array}$ & 136 & & & & \\
\hline 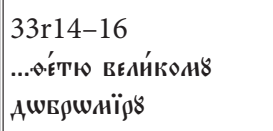 & 137 & & & & \\
\hline 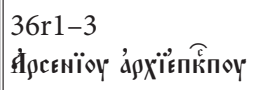 & 138 & & & & \\
\hline $\begin{array}{l}36 r 4-5 \\
\text { †̈́сифоу новоноу } \\
\text { йспов'ддникоу }\end{array}$ & 139 & & & & \\
\hline 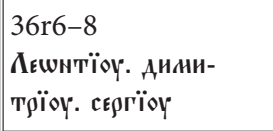 & 140 & & & & \\
\hline 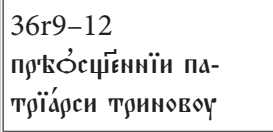 & & & & & \\
\hline $\begin{array}{l}\text { 36r10-12 } \\
\text { †ша́киноу пртвоноу }\end{array}$ & 141 & & & & \\
\hline
\end{tabular}


Table 1 (cont.)

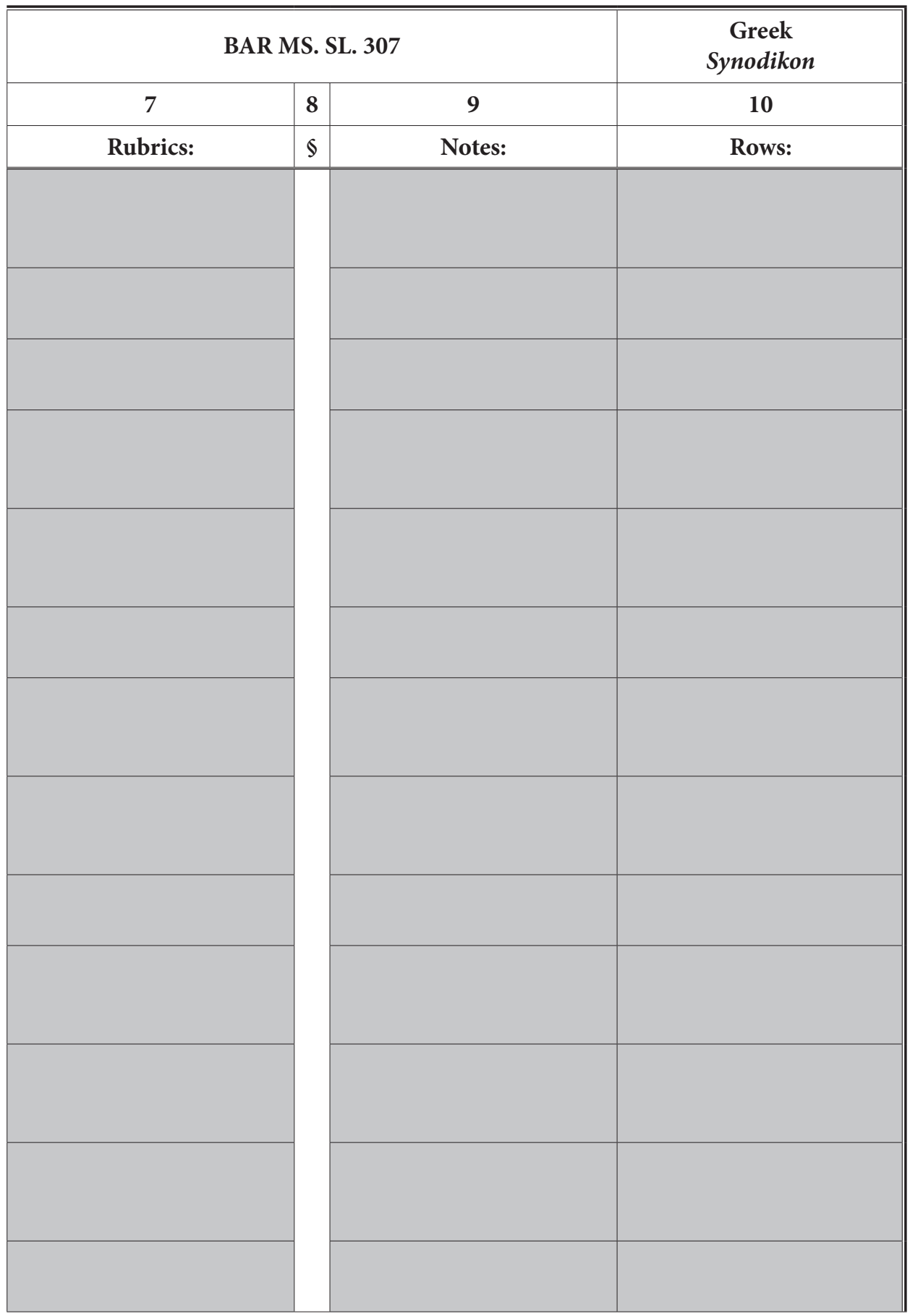




\begin{tabular}{|c|c|c|c|c|c|}
\hline \multicolumn{3}{|c|}{ Palauzov's copy } & \multicolumn{3}{|c|}{ Drinov's copy } \\
\hline 1 & 2 & 3 & 4 & 5 & 6 \\
\hline Rubrics: & $\$$ & Notes: & Rubrics: & $\S$ & Notes: \\
\hline $\begin{array}{l}\text { 36r13-15 } \\
\text { Василїоу. ішакиндоу. } \\
\text { ігнатїв. }\end{array}$ & 142 & & & & \\
\hline $\begin{array}{l}\text { 36r16-18 } \\
\text { Макарїоу Т९ЪЕАА- } \\
\text { ЖЕнНОАоу }\end{array}$ & 143 & & & & \\
\hline 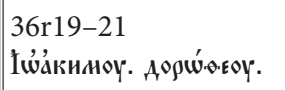 & 144 & & & & \\
\hline $\begin{array}{l}\text { 36v1-2 } \\
\text { Iwáникїв }\end{array}$ & 145 & & & & \\
\hline $\begin{array}{l}\text { 36v3-4 } \\
\text { G̈̈нешноу }\end{array}$ & 146 & & & & \\
\hline 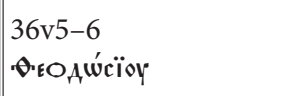 & 147 & & & & \\
\hline $\begin{array}{l}\text { 36v7-8 } \\
\text { Iӝаникїоу }\end{array}$ & 148 & & & & \\
\hline 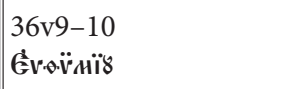 & 149 & & & & \\
\hline 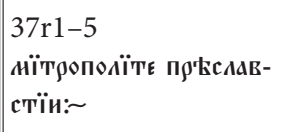 & 150 & & & & \\
\hline 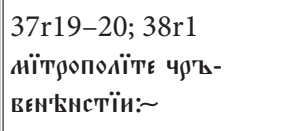 & 151 & & & & \\
\hline $\begin{array}{l}\text { 37v6-8 } \\
\text { нїтрополїте А०- } \\
\text { вєчьстїИ:- }\end{array}$ & 152 & & & & \\
\hline 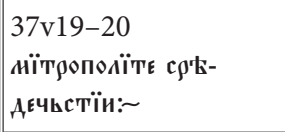 & 153 & & & & \\
\hline $\begin{array}{l}\text { 38r11-12 } \\
\text { нї̈трополїте } \\
\text { о́вєчьстїи+ }\end{array}$ & 154 & & & & \\
\hline 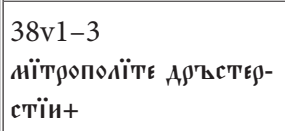 & 155 & & & & \\
\hline
\end{tabular}


Table 1 (cont.)

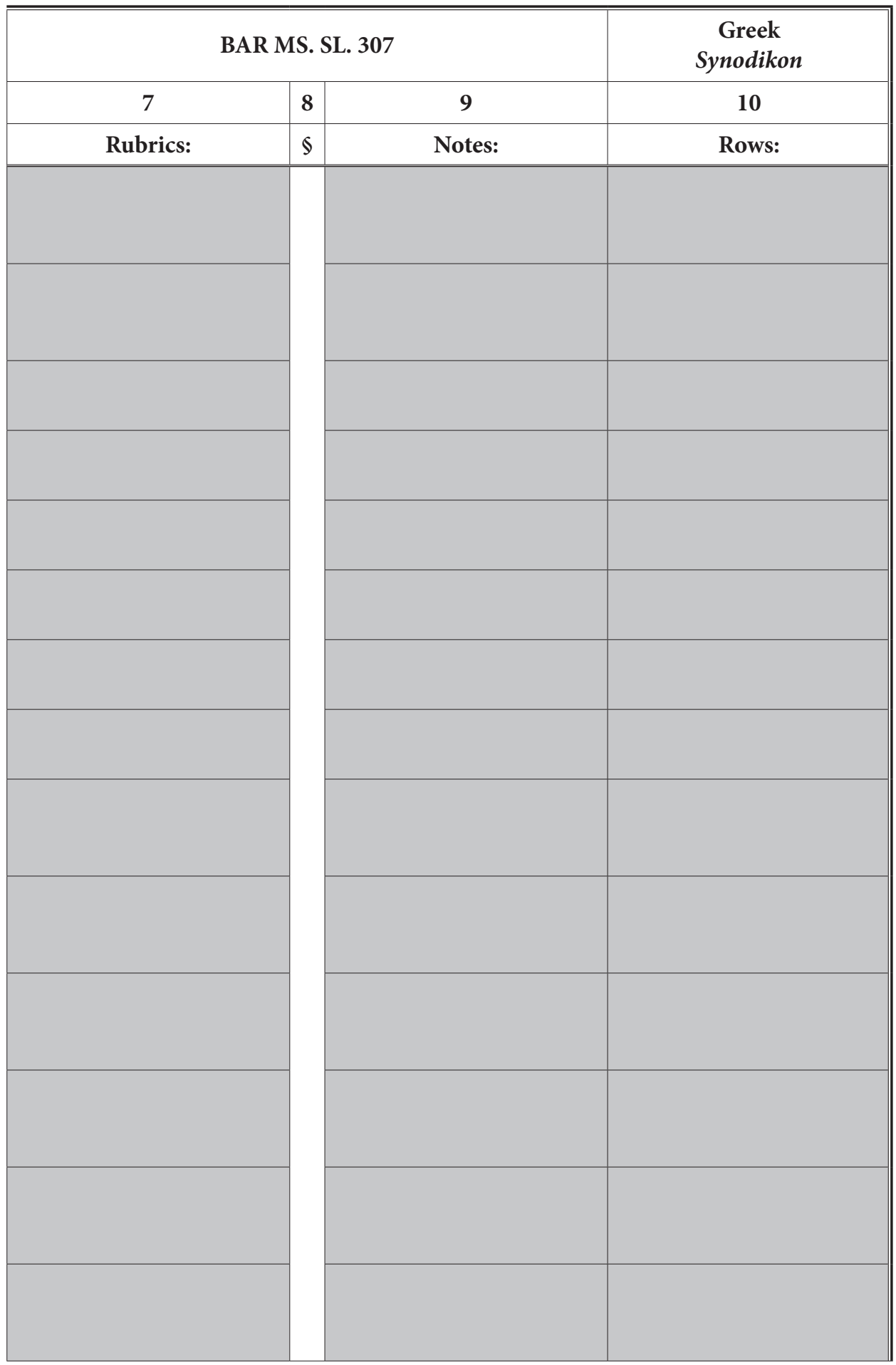




\begin{tabular}{|c|c|c|c|c|c|}
\hline \multicolumn{3}{|c|}{ Palauzov's copy } & \multicolumn{3}{|c|}{ Drinov's copy } \\
\hline 1 & 2 & 3 & 4 & 5 & 6 \\
\hline Rubrics: & $\$$ & Notes: & Rubrics: & $\S$ & Notes: \\
\hline 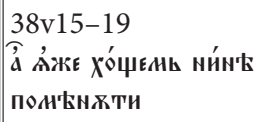 & 155 & & & & \\
\hline $\begin{array}{l}\text { 38v20-39r1 } \\
\text { Янттшнїє. данїиль. }\end{array}$ & 156 & & & & \\
\hline 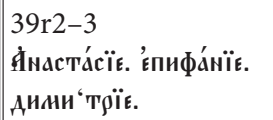 & 157 & & & & \\
\hline 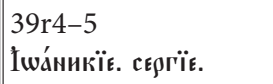 & 158 & & & & \\
\hline 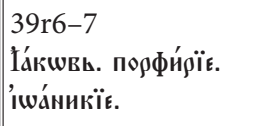 & 159 & & & & \\
\hline $\begin{array}{l}\text { 39r8-10 } \\
\text { Gа́ва. Фєода́сї̈. } \\
\text { диниттё̈в }\end{array}$ & 160 & & & & \\
\hline \begin{tabular}{||l} 
39r11-12 \\
Кӥрнль. дїшнисїв.
\end{tabular} & 161 & & & & \\
\hline $\begin{array}{l}\text { 39r13-15 } \\
\text { GТЕФАНЬ. КАИАЕнТТ. } \\
\text { ІІШСИФь. }\end{array}$ & 162 & & & & \\
\hline $\begin{array}{l}\text { 39r16-17 } \\
\text { G̈̈нЕшнь Аит९опо- } \\
\text { Анть }\end{array}$ & 163 & & & & \\
\hline $\begin{array}{l}\text { 39r18-21 } \\
\text { Василї̈. ма́рко. } \\
\text { никодиннь. }\end{array}$ & 164 & & & & \\
\hline 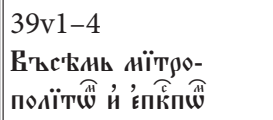 & 165 & & & & \\
\hline 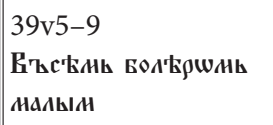 & 166 & & & & \\
\hline 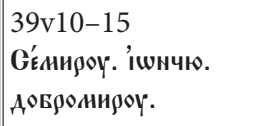 & 167 & & & & \\
\hline
\end{tabular}


Table 1 (cont.)

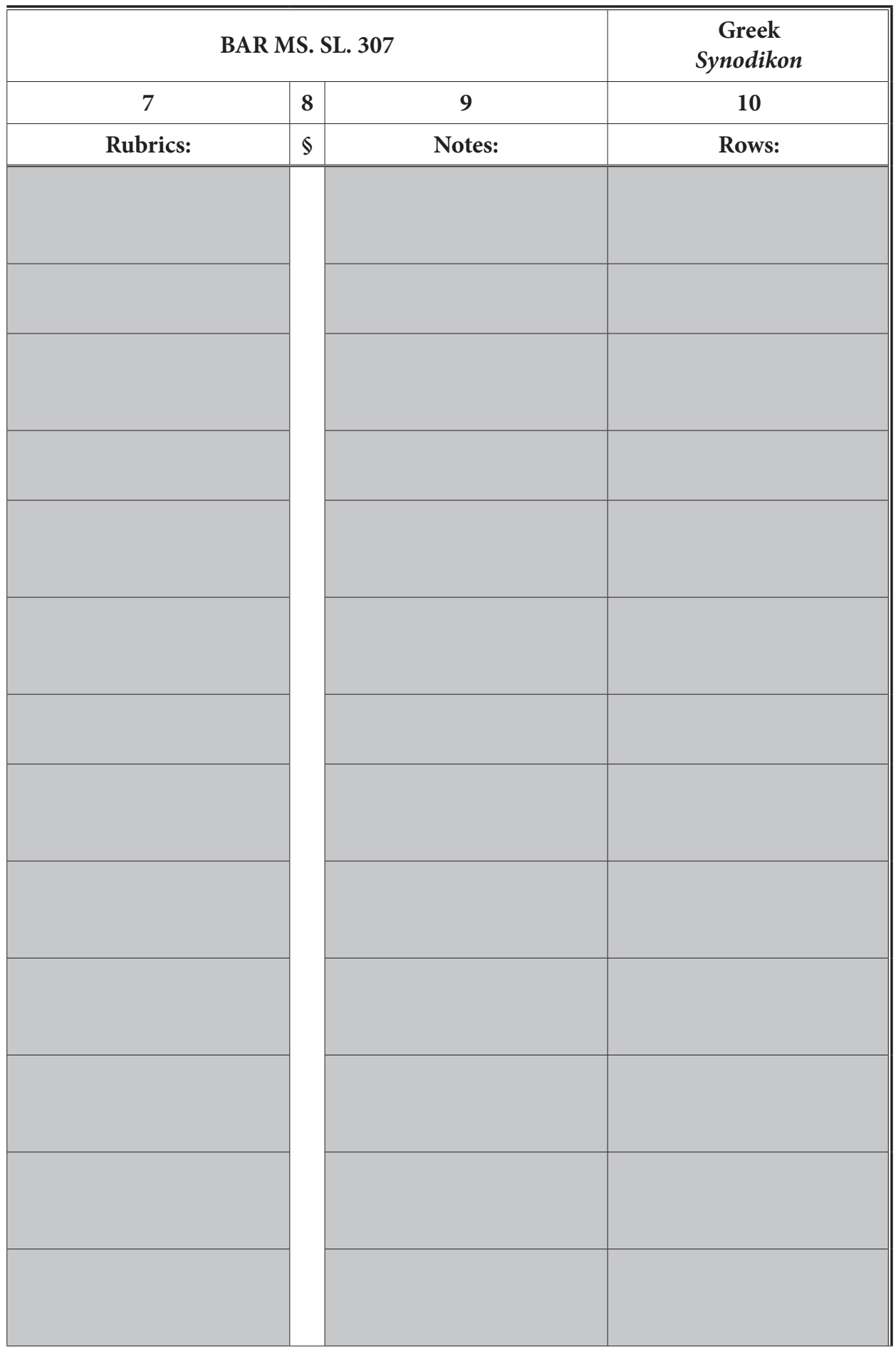




\begin{tabular}{|c|c|c|c|c|c|}
\hline \multicolumn{3}{|c|}{ Palauzov's copy } & \multicolumn{3}{|c|}{ Drinov's copy } \\
\hline 1 & 2 & 3 & 4 & 5 & 6 \\
\hline Rubrics: & $\S$ & Notes: & Rubrics: & $\S$ & Notes: \\
\hline 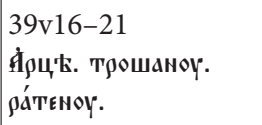 & 168 & & & & \\
\hline 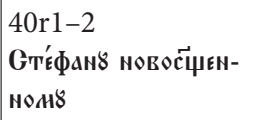 & 169 & & & & \\
\hline 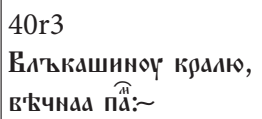 & 170 & & & & \\
\hline $\begin{array}{l}\text { 40r4 } \\
\text { АЕспОтю ОЙГАЕШОY, }\end{array}$ & 171 & & & & \\
\hline 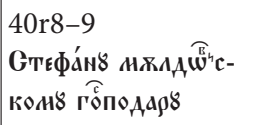 & 172 & & & & \\
\hline 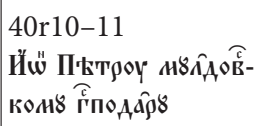 & 173 & & & & \\
\hline 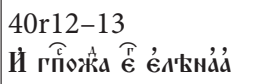 & 174 & & & & \\
\hline 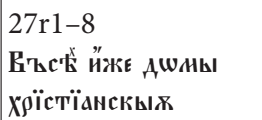 & 175 & & & & \\
\hline $\begin{array}{l}\text { 27r10-27v6 } \\
\text { Якиндина варладана. }\end{array}$ & 176 & & & & \\
\hline 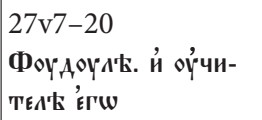 & 177 & & & & \\
\hline
\end{tabular}

Description: Columns 1, 4, 7 contain the incipits of the rubrics in Palauzov's (P), Drinov's (D) and Romanian $(\mathrm{R})$ versions, taking into account the real segmentation according to the initial and red letters. The new edition of the Synodikon of tsar Boril as well as the edition of the Palaeologan Synodikon reflect the same segmentation. Columns 2 and 5 show the paragraphs in the edition by Popruženko (М.Г. Попруженко, Синодикъ изаря Борила...), while column 8 shows the paragraphs in R, thus linking the new editions with the edition by Popruženko. 
Table 1 (cont.)

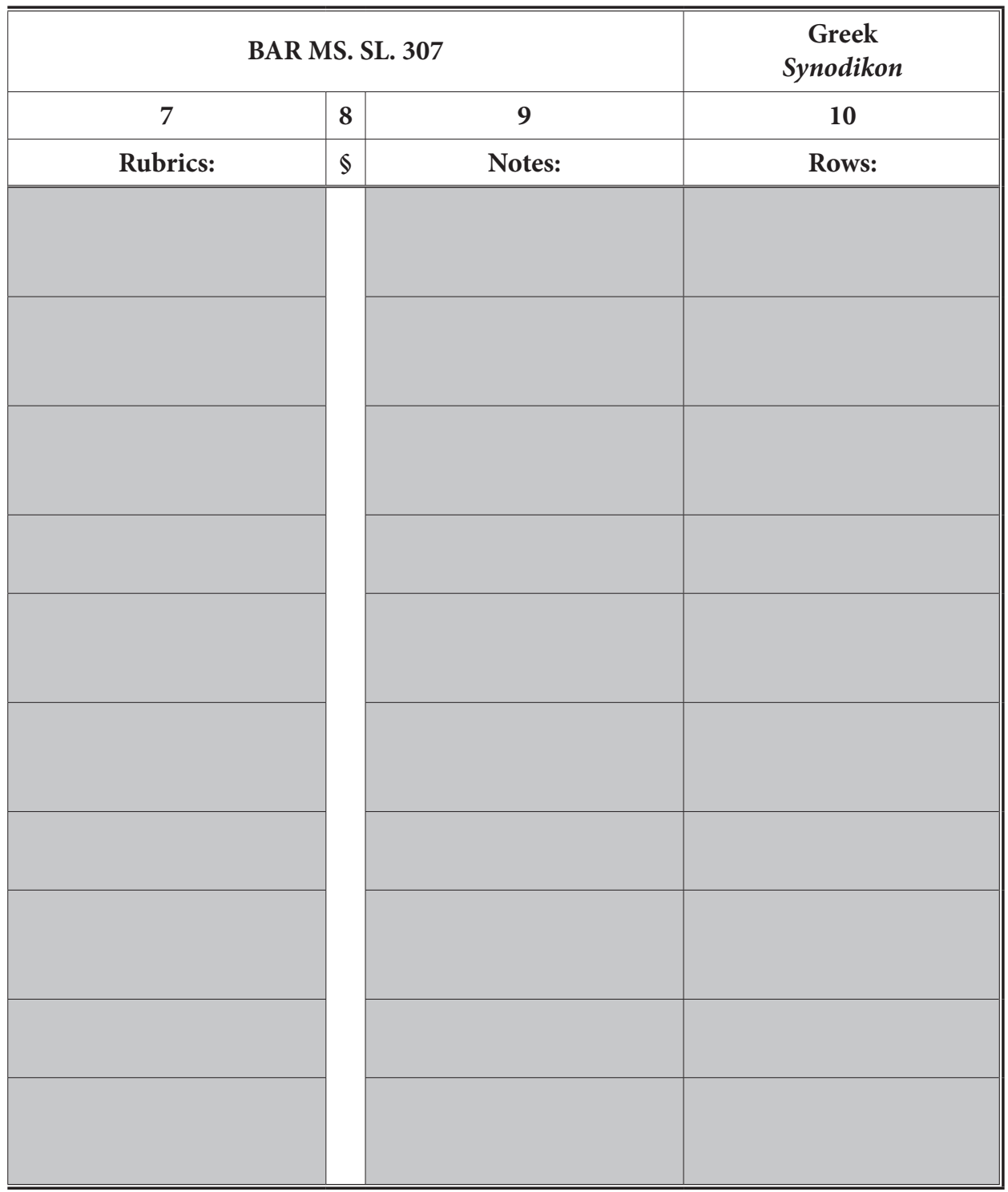

Columns 3, 6, 9 contain comments on the order of the rubrics in the three versions, on differences in text segmentation or on missing and damaged parts in the rubrics themselves. The last column shows the Greek source. Correspondences to the Greek Synodikon according to the edition of Gouillard are marked with G.; the Horos of the Synod of 843 is introduced by G., followed by the page number in the same edition. The Letter of Patriarch Cosmas is reported as Patriarch Cosmas. Other sources are reported as follows: A. Rigo, L'Assemblea generale... is the source of the Holy

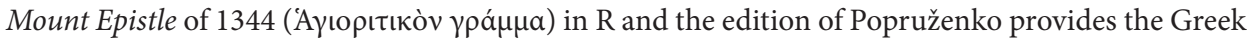
text of the anathema ( $\$ 16$ in $\mathrm{D}$ and $\mathrm{R}$ ), which is preserved in the printed Greek triodia. 


\section{Bibliography}

\section{Sources}

Anne Comnène, Alexiade, vol. III, ed. B. Leib, Paris 1945, ${ }^{2} 1967$.

Božilov I., Totomanova A., Biljarski I., Borilov sinodik. Izdanie i prevod, Sofija 2010.

Gouillard J., Le Synodikon de l'Orthodoxie: édition et commentaire, "Travaux et mémoires du Centre de recherches d'histoire et civilisation byzantines” 2, 1967, p. 1-316.

Mošın V.A., Serbskaja redakcija sinodika v nedelju pravoslavija. Teksty, "Византийский временник" / "Vizantijskij vremennik" 17, 1960, p. 278-353.

Synodicum Bulgaricum 1211, ed. A. Totomanova, [in:] The Great Councils of the Orthodox Churches. From Constantinople 861 to Moscow 2000, ed. A. Melloni, vol. I, Turnhout 2016 [= CC.COGD 4.1], p. 426-468.

\section{Secondary Literature}

BilJarski I., Paleologovijat sinodik v slavjanski prevod, Sofija 2013.

Biljarski I., Cibranska-Kostova M., Za edin kompoziten tip i za Paleologovija wariant na slavjanskija Sinodik v Nedeljata na pravoslavieto, "Palaeobulgarica" 36.1, 2012, p. 51-65.

BožILov I., Vizantijskijat svjat, Sofija 2008.

Gouillard J., Une source grecque du Synodik de Boril: la lettre du patriarche Cosmas, "Travaux et mémoires du Centre de recherches d'histoire et civilisation byzantines" 4, 1970, p. 361-374.

Minczew G., Borilovijat sinodik 800 godini po-kăsno, "Palaeobulgarica” 35.2, 2011, p. 68-84.

Mošın V.A., Serbskaja redakcija sinodika v nedelju pravoslavija. Analiz tekstov, “Византийский временник" / "Vizantijskij vremennik" 16, 1959, p. 317-394.

Popruženko M.G., Sinodik carja Borila, “Български старини” / “Bălgarski starini” 8, 1928.

Rigo A., L'Assemblea generale atonita del 1344 su un gruppo di monaci bogomili (ms. Vat.Gr. 604 ff. 11r-12v), "Cristianesimo nella Storia. Ricerche storiche, esegetiche, teologiche" 5, 1984, p. $475-506$.

Rigo A., Monaci esicasti e monaci bogomili. Le accuse di messalianismo e bogomilismo rivolte agli isicasit ed il problema dei rapport tra esicasmo e bogomislismo, Firenze 1989 [= OV 2].

Stefanov P., Nov pogled kăm unijata meždu Bălgarskata i Rimskata cărkva prez XIII v., "Преславска книжовна школа" / "Preslavska knižovna škola” 5, 2001, p. 343-352.

Toтomanova A., Ezikăt na XIV v. i săstavăt na Palauzovija sbornik, "Palaeobulgarica” 36.1, 2012, p. 24-37.

Totomanova A., Sinodik carja Borila v sbornike Palauzova (NBKM № 289), [in:] XXI ežegodnaja bogoslovskaja konferencija. Cerkovno-istoričeskie issledovanija $v$ kontekste sovremennoj nauki, Moskva 2011, p. 165-171.

Totomanova A., Za edna paronomazija v Borilovija sinodik, [in:] Slovesa prěčjud’naja. Jubileen sbornik v čest na prof. Ivan Bujukliev, ed. A. Totomanova, R. Vlahova-Rujkova, Sofija 2012, p. 36-43. 


\begin{abstract}
The paper compares the content and the structure of the three extant South Slavonic Synodika: Boril's Synodikon as preserved in the so-called Palauzov copy of the $14^{\text {th }}$ century (НБКМ № 289); Drinov's Synodikon (НБКМ № 432), previously considered to be a $16^{\text {th }}$ century copy of Boril's Synodikon, and the recently published South Slavonic Synodikon from the $16^{\text {th }}$ century, kept in the library of the Romanian Academy of Sciences (BAR MS. SL. 307). The comparison is supported by a table showing the rubrics and their order in the three Synodika. It demonstrates that while Boril's Synodikon is based on a translation of Comnenian version of the Synodikon of Orthodoxy, and while the South Slavonic Synodikon from Romania must be unequivocally attributed to the later Palaeologan version of the Greek text, the so-called Drinov copy represents a compilation of Boril's Synodikon in its $14^{\text {th }}$ version and the Palaeologan Synodikon. In fact, Drinov's Synodikon contains all of the important interpolations and insertions of Boril's Synodikon related to specifically Bulgarian circumstances and history, ranging from anti-Bogomilist anathemas to a list of Bulgarian rulers (comprising two historical accounts as well). Its initial part, however, follows the Palaelogan text preserved in BAR MS. SL. The unknown compiler obviously targeted a Bulgarian audience; in all likelihood, he was Bulgarian himself. Some textological features common to both Drinov's and Palaelogan Synodikon suggest that the translated part of Drinov's Synodikon and the Romanian Synodikon must have had a common antigraph. The latter fact allows us to conclude that the translation of the Palaeologan version of the Synodikon of Orthodoxy is an integral part of the tradition of the Bulgarian Synodikon; the presumed common antigraph was written in Bulgarian Tărnovo orthography, traces of which are found in Drinov's text. As to the location of this translation, we can only speculate that it might have been completed in a monastic centre different than Tărnovo by the end of the $14^{\text {th }}$ century.
\end{abstract}

Keywords: Synodikon of Orthodoxy, Palaeologan and Comnenian versions of the Synodikon, Bulgarian translations and versions of the Synodikon.

Anna-Maria Totomanova

St. Clement of Ohrid University of Sofia 15 Tsar Osvoboditel blvd. 1000 Sofia, Bulgaria atotomanova@abv.bg 